\title{
Hamiltonian Renormalisation I: Derivation from Osterwalder-Schrader Reconstruction
}

\author{
T. Lang $\stackrel{1 *}{,}$ K. Liegener ${ }^{1 \dagger}$, T. Thiemann ${ }^{1 \ddagger}$ \\ ${ }^{1}$ Inst. for Quantum Gravity, FAU Erlangen - Nürnberg, \\ Staudtstr. 7, 91058 Erlangen, Germany
}

July 9, 2019

\begin{abstract}
A possible avenue towards a non-perturbative Quantum Field Theory (QFT) on Minkowski space is the constructive approach which employs the Euclidian path integral formulation, in the presence of both ultraviolet (UV) and infrared (IR) regulators, as starting point. The UV regulator is to be taken away by renormalisation group techniques which in case of success leads to a measure on the space of generalised Euclidian fields in finite volume. The removal of the IR regulator corresponds to the thermodynamic limit of the system in the statistical physics sense. If the resulting measure obeys the Osterwalder-Schrader axioms, the actual QFT on Minkowski space is then obtained by Osterwalder-Schrader reconstruction.

In this work we study the question whether it is possible to reformulate the renormalisation group non-perturbatively directly at the operator (Hamiltonian) level. Hamiltonian renormalisation would be the natural route to follow if one had easier access to an interacting Hamiltonian operator rather than to a path integral, at least in the presence of UV and/or IR cut-off, which is generically the case in complicated gauge theories such as General Relativity.

Our guiding principle for the definition of the direct Hamiltonian renormalisation group is that it results in the same continuum theory as the covariant (path integral) renormalisation group. This makes the present work different from other approaches to Hamiltonian renormalisation. In order to achieve this, we invert the Osterwalder-Schrader reconstruction, which may be called Osterwalder-Schrader construction of a Wiener measure from the underlying Hamiltonian theory. The resulting correspondence between reflection positive measures and Osterwalder-Schrader data consisting of a Hilbert space, a Hamiltonian and a ground state vector allows us to monitor the effect of the renormalisation flow of measures in terms of their Osterwalder-Schrader data which motivates a natural direct Hamiltonian renormalisation scheme.
\end{abstract}

*thorsten.lang@gravity.fau.de

${ }^{\dagger}$ klaus.liegener@gravity.fau.de

${ }^{\ddagger}$ thomas.thiemann@gravity.fau.de 


\section{Contents}

1 Introduction $\quad 4$

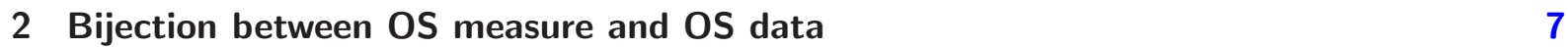

2.1 Preliminaries . . . . . . . . . . . . . . . . . . . . . 8

2.2 Constructing the OS measure from OS data . . . . . . . . . . . . . . . . 10

2.3 Constructing the OS data form the OS measure . . . . . . . . . . . . . . . . . . 14

2.4 OS data reconstruction and OS measure construction are inverses . . . . . . . . . 16

2.4 .1 Reproduction of OS data . . . . . . . . . . . . . . . . . 16

2.4.2 Reproduction of the OS measure . . . . . . . . . . . . . . . 17

$\begin{array}{lll}3 & \text { Brief review of path integral renormalisation } & 18\end{array}$

4 Hamiltonian renormalisation $\quad 22$

4.1 Derivation of the Path Integral Induced Hamiltonian Renormalisation Scheme . . . . 24

4.2 Motivation of the Direct Hamiltonian Renormalisation Scheme . . . . . . . . . . . . 27

5 Outlook: Hamiltonian Renormalisation of Generally Covariant Theories 30

$\begin{array}{ll}\text { A Inductive Limits } & 31\end{array}$

B Notes on Nelson-Symanzik Positivity 32 


\section{Notation:}

In this paper we will deal with quantum fields in the presence of an infrared cut-off $R$ and with smearing functions of finite time support in $[-T, T]$. The spatial ultraviolet cut-off is denoted by $M$ and has the interpretation of the number of lattice vertices in each spatial direction. We will mostly not be interested in an analogous temporal ultraviolet cut-off $N$ but sometimes refer to it for comparison with other approaches. These quantities allow us to define dimensionful cut-offs $\epsilon_{R M}=\frac{R}{M}, \delta_{T N}=\frac{T}{N}$. In Fourier space we define analogously $k_{R}=\frac{2 \pi}{R}, k_{M}=\frac{2 \pi}{M}, k_{T}=\frac{2 \pi}{T}, k_{N}=\frac{2 \pi}{N}$.

We will deal with both instantaneous fields, smearing functions and Weyl elements as well as corresponding temporally dependent objects. The instantaneous objects are denoted by lower case letters $\phi_{R M}, f_{R M}, w_{R M}\left[f_{R M}\right]$, the temporally dependent ones by upper case ones $\Phi_{R M}, F_{R M}, W_{R M}\left[f_{R M}\right]$. As we will see, smearing functions $F_{R M}$ with compact and discrete (sharp) time support will play a more fundamental role for our purposes than those with a smoother dependence.

Osterwalder-Schrader reconstruction concerns the interplay between time translation invariant, time reflection invariant and reflection positive measures (OS measures) $\mu_{R M}$ on the space of history fields $\Phi_{R M}$ and their corresponding Osterwalder-Schrader (OS) data $\mathcal{H}_{R M}, \Omega_{R M}, H_{R M}$ where $\mathcal{H}_{R M}$ is a Hilbert space with cyclic (vacuum) vector $\Omega_{R M}$ annihilated by a self-adjoint Hamiltonian $H_{R M}$. Together, the vector $\Omega_{R M}$ and the scalar product $\langle., .\rangle_{\mathcal{H}_{R M}}$ define a measure $\nu_{R M}$ on the space of instantaneous fields $\phi_{R M}$.

Renormalisation consists in defining a flow or sequence $n \rightarrow \mu_{R M}^{(n)}, n \in \mathbb{N}_{0}$ for all $M$ of families of measures $\left\{\mu_{R M}^{(n)}\right\}_{M \in \mathbb{N}}$. The flow will be defined in terms of a coarse graining or embedding map $I_{R M \rightarrow M^{\prime}}, M<M^{\prime}$ acting on the smearing functions and satisfying certain properties that will grant that 1 . the resulting fixed point family of measures, if it exists, is cylindrically consistent and 2 . the flow stays within the class of OS measures. Fixed point quantities are denoted by an upper case *, e.g. $\mu_{R M}^{*}$. 


\section{Introduction}

Renormalisation in Quantum Field Theory (QFT) comes in two flavours, namely perturbative and non-perturbative schemes. In the perturbative formulation it is a matter of taste whether one uses operator or path integral methods as both are based on the underlying free theory for which there is a precise bijection between both methods. Roughly speaking, the path integral of a free theory can be obtained by Trotter product techniques from the operator formalism (Feynman-Kac formula). Conversely, the operator formalism is recovered from the path integral of the free theory by Osterwalder-Schrader reconstruction.

On the other hand, non-perturbative approaches, at least in 4 dimensions, are almost entirely based on the path integral approach, in particular in its Euclidian formulation following the constructive QFT programme [1-4]. The reason for this is that in the presence of both ultraviolet (UV) and infrared (IR) cut-off, it is very easy to postulate a rigorously defined path integral for the interacting theory as the system retains only a finite number of degrees of freedom and at least for QFT on Minkowski space a path integral defined by the Lebesgue measure on the space of those finitely many degrees of freedom times the exponential of minus the Euclidian action is a well motivated starting point. The challenge is then to show that one can take first the UV and then the IR regulator away, resulting in a rigorously defined Euclidian measure obeying the Osterwalder-Schrader axioms, most prominently reflection positivity [5-7]. The IR cut-off removal corresponds to the thermodynamic limit in the statistical physics sense which is what Euclidian QFT is and is usually less problematic than the UV cut-off removal.

The technical tool for the UV cut-off removal is the renormalisation programme, which we will review, in the Euclidian setting, for a rather general theory in a subsequent section. In our terminology, a QFT with a short distance or finite resolution cut-off is simply a one parameter family of more or less motivated probability measures $\epsilon \mapsto \mu_{\epsilon}^{(0)}$ that typically arise by ad hoc discretisations on finite lattices of finite resolution $\epsilon$. Here $\epsilon$ may be defined via a background metric or not, in the presence of an IR cut-off the spacetime available to the quantum field is compact and hence $\epsilon$ can be defined background independently by counting the number of degrees of freedom. It is important to note, that the ad hoc prescription for building $\mu_{\epsilon}^{(0)}$, while generically plagued by ambiguities ("couplings" on which the measure depends parametrically) is the same for all $\epsilon$. The measure $\mu_{\epsilon}^{(0)}$ is supposed to produce all Schwinger functions from the generating functional $\mu_{\epsilon}^{(0)}\left(\exp \left(i \Phi_{\epsilon}\left[F_{\epsilon}\right]\right)\right)$ by (functional) derivation at $F_{\epsilon}=0$ with respect to $F_{\epsilon}$. Here $\Phi_{\epsilon}$ is the discretised field at cut-off $\epsilon$ over which $\mu_{\epsilon}^{(0)}$ integrates and $F_{\epsilon}^{\prime}$ is the restriction to the lattice vertices of a continuum smearing function $F_{\epsilon}$ which however only resolves scales at or above $\epsilon$. In terms of the undiscretised or continuum field $\Phi$ one has the relation $\Phi\left[F_{\epsilon}\right]=: \Phi_{\epsilon}\left[F_{\epsilon}^{\prime}\right]$ between the smeared fields. As we will see, the details of the relation between $F_{\epsilon}$ and $F_{\epsilon}^{\prime}$ is what defines a block spin or better coarse graining transformation. If one had a continuum measure $\mu$ integrating over continuum fields $\Phi$, then one would identify the generating functional at $\epsilon$ with $\mu\left(\exp \left(i \Phi\left(F_{\epsilon}\right)\right)\right.$. In other words, the $\mu_{\epsilon}^{(0)}$ would define the cylindrical projections of $\mu$. However, for this to be the case, the family $\mu_{\epsilon}^{(0)}$ must be cylindrically consistent: We can view $F_{\epsilon}$ as a special case of a function $\tilde{F}_{\epsilon^{\prime}}$ at a scale $\epsilon<\epsilon^{\prime}$, that is, $F_{\epsilon}=\tilde{F}_{\epsilon^{\prime}}$ as functions in the continuum. They will give rise to finite resolution functions $F_{\epsilon}^{\prime}, \tilde{F}_{\epsilon^{\prime}}^{\prime}$. Now by construction the continuum measure will assign to both functions the same generating functional but that is not automatically the case for the generating functionals in terms of the family $\mu_{\epsilon}^{(0)}$.

Renormalisation now consists in constructing a sequence $\mu_{\epsilon}^{(n)}$ of measure families where one obtains $\mu_{\epsilon}^{(n+1)}$ from $\mu_{\epsilon / 2}^{(n)}$ by integrating out the degrees of freedom at scale $\epsilon / 2$ that do not contribute to scale $\epsilon$. If the sequence converges or at least has a fixed point (accumulation point) family $\mu_{\epsilon}^{*}$ then by construction that family is consistent. Then the usual extension theorems [9] can be consulted in order to show that the family extends to a continuum measure $\mu^{*}$. One then must show that $\mu^{*}$ obeys the Osterwalder-Schrader axioms. To be a viable physical theory, all but a finite number of the ambiguous parameters (the so-called relevant ones), that parametrise the original family $\mu_{\epsilon}^{(0)}$, must have been fixed pointed during the renormalisation process. All of this will be made precise during 
the course of the paper.

How does the Hamiltonian description fit into this picture? We restrict the block spin transformations defining the renormalisation flow by the requirement that the measures $\mu_{\epsilon}^{(n)}$ for each $n \in \mathbb{N}_{0}, \epsilon>0$ satisfy at least a subset of the Osterwalder-Schrader axioms sufficient in order to reconstruct quantum theory (see e.g. [10] for such a subset). This will impose a certain restriction on the choice of the initial family $\mu_{\epsilon}^{(0)}$ as well as the choice of coarse graining map. Under these circumstances we obtain for each $n, \epsilon$ a triple $\left(\mathcal{H}_{\epsilon}^{(n)}, H_{\epsilon}^{(n)}, \Omega_{\epsilon}^{(n)}\right)$ consisting of a Hilbert space, a self-adjoint and positive semi-definite Hamiltonian operator thereon and a cyclic vacuum vector which is a zero eigenvector for the Hamiltonian. We will call them Osterwalder-Schrader (OS) data in what follows. Therefore, from the measure theoretic perspective, Hamiltonian renormalisation consists in precisely the flow of this family of triples. Its fixed point $\left(\mathcal{H}^{*}, H^{*}, \Omega^{*}\right)$ are the Osterwalder-Schrader data for $\mu^{*}$ provided that the measure family has a fix point $\mu^{*}$ satisfying the Osterwalder-Schrader (OS) axioms.

In this paper we ask the natural question, whether the renormalisation sequence of OS data really has to be constructed via the measure theoretic framework or whether there is a more direct route that remains entirely within the Hamiltonian setting. In fact, the initial measure family $\mu_{\epsilon}^{(0)}$ can only be "guessed" for a tiny subset of theories that are of the form: Lebesgue measure times exponential of minus the negative of the classical Euclidian action. This is the subset of theories for which the classical Hamiltonian is 0 . not constrained to vanish, 1. a quadratic polynomial in the bosonic canonical momenta with coefficients that are 2. independent of the configuration field and 3. independent of time. If the zeroth assumption fails to hold, then we consider a generally covariant theory that is spacetime diffeomorphism invariant and the measure will typically receive very complicated corrections from the gauge fixing Dirac-bracket determinant (Fadeev-Popov determinant), see e.g. [11-13]. If the first condition is not met, then the momenta cannot even be integrated out and one must use a path integral formulation which is based on a measure on a history phase space rather than history configuration space [11]. If the second condition is violated, one can integrate out the momenta, but the Lebesgue measure receives a non-trivial Jacobian. Finally, if the third assumption is false, then a Wick rotation to imaginary time is generically impossible. This is the case of QFT in curved spacetime since general background spacetime metrics do not admit a gauge (coordinate system) in which the metric is analytic in the coordinates. In this paper we will deal with theories that underlie restriction 1 . and 3. but not necessarily the zeroth and second. This includes quantum gravity coupled to standard matter when suitable gauge fixing of spacetime diffeomorphism invariance employing matter reference systems is in place [14]. This works, because in that case the metric is a dynamical field with no explicit time dependence. More generally then, we restrict attention to conservative systems (which is no actual restriction, every system becomes conservative when including the background as a dynamical field) where possible diffeomorphism invariance has been gauge fixed.

Given such a set-up, the construction of an initial family of triples consisting of Hilbert space, Hamiltonian and vacuum is relatively straightforward in this setting. If we are interested in the continuum OS data, then the route via the construction of the initial measure family and its renormalisation looks like a very indirect and complicated approach that one would like to circumvent. In order to perform Hamiltonian renormalisation directly without using the OS measure, it is necessary to understand how the bijection between the OS measure and the OS data actually works. In [10], the part of the proof, usually given for Minkowski space QFT, that suffices to reconstruct the OS data from the measure, has been adapted to a more general setting including generally covariant QFT. In this paper we will develop the opposite direction and show that both maps are inverses of each other when the theory has been suitably formulated. After having performed this step we can try to define Hamiltonian renormalisation directly without recourse to the functional integral in such a way that both renormalisation procedures are mapped into each other. We will be only partially successful in doing so: While we can show that for suitable coarse graining maps the path integral flow stays in the class of reflection positive measures, the construction of these measures cannot be entirely avoided. On the other hand, the framework suggests a natural modification of 
the path integral induced flow giving rise to a direct flow of the OS data without using path integrals which we will demonstrate to have the same fixed points in the case of free field theories. In that sense we differ from earlier, related suggestions in the literature (see e.g. [15-32] or for applications to quantum gravity [33-40] and references therein). Rather, our approach is closer in spirit to a purely Hamiltonian scheme similar to the frameworks developed by Glazek and Wilson [41] building on Wegner [42] for which there are vast applications in the literature (see e.g. $[43,44]$ and references therein for more recent applications within perturbative interacting QFT and quantum mechanics). Our considerations differ from these latter works not only in that the relation to the path integral renormalisation scheme remains more transparent. Specifically, our renormalisation group flow will be in terms of families of isometric injections from Hilbert spaces of lower to those of higher resolution rather than block diagonalising unitaries with respect to higher and lower energy modes. Moreover, our formulation is intrinsically background independent and non perturbative.

Roughly speaking, direct Hamiltonian renormalisation is parametrised by a family of spatial coarse graining maps on the space of test functions. These in turn define embeddings of coarse grained Hilbert spaces. The flow of the OS data is such that these embeddings are isometric, in particular when the fixed point is reached. The continuum Hilbert space is then the corresponding inductive limit, the vacua are mapped into each other by those isometries. The flow of the Hamiltonians is such that they annihilate the corresponding flow of vacua, however, the continuum Hamiltonian defined by the fixed point family is not the inductive limit of the coarse grained Hamiltonians. Indeed, for an inductive family of Hamiltonian operators it is necessary that the family of isometric injections is equivariant with respect to the family of Hamiltonians, however, we obtain only a weaker version of that.

This is because the fixed point family only defines a consistently defined quadratic form (if certain universality properties with respect to the chosen coarse graining map are satisfied). Such a quadratic form does not necessarily define an operator. By contrast, the inductive limit construction grants the existence of such a Hamiltonian but would require that the Hamiltonians preserve the image of the injections which again would be counter intuitive. This also means that we are not in conflict with Haag's theorem which for an infinite number of degrees of freedom predicts difficulties when trying to define different Hamiltonians on the same Hilbert space. On the other hand, the fact that an interacting vacuum vector is constructed in the sense that the Hamiltonian quadratic form has vanishing matrix element with any other state increases the chance that the quadratic form in fact extends to an operator. Moreover, if the initial operator family is positive and symmetric, then the flow preserves this property and thus results in a positive, symmetric quadratic form. This quadratic form then, provided it is closable, has at least the Friedrichs extension as a self-adjoint operator. Of course, it is then a physics question if that self-adjoint extension is viable.

We also note that in order to construct the Hamiltonian from the OS measure we need that time is continuous as the Hamiltonian generates the corresponding OS contraction semi-group. Hence, the measure renormalisation that we consider here is one where time is always continuous. This asymmetric treatment of time and space is mandatory if we want to construct the Hamiltonian.

The architecture of this paper is as follows:

In section 2 we derive the bijection between OS measure and OS data.

In section 3 we formulate measure renormalisation in a language that slightly differs from the usual one and that is geared towards Hamiltonian renormalisation in our sense. We present the dictionary between the usual language and our own.

In section 4 we derive path integral induced Hamiltonian renormalisation from first principles, that is, using the language of sections 2 and 3. The main input is the coarse graining map. We motivate the choice of physically acceptable maps starting from the classical theory where the role of the corresponding isometric injections of the quantum theory is played by classical partial symplectomorphisms. This is in accordance with Kijowski's programme of formulating large quantum 
theories as consistent families of small ones [45-48]. This also makes it plausible that two choices of coarse graining maps result in unitarily equivalent QFT's. The Hamiltonians that one obtains via OS reconstruction from the path integral flow have the counter intuitive property to commute with the projections onto the coarse grained Hilbert spaces. We uncover the semigroup law of the OS contraction semigroups generated by these Hamiltonians as the reason responsible for that feature. This observation lies at the heart of defining a closely related direct Hamiltonian flow which does not have that counter intuitive feature.

In section 5 we summarise our findings and discuss future applications of the framework developed in the present paper, in particular for theories that are generally covariant and thus background independent.

In appendix A we recall elementary notions from the theory of inductive limits in our language for the benefit of the reader.

In appendix B we supply some background material on criteria when a Hamiltonian contraction semigroup defines a Wiener measure.

This paper is the first in a series of four papers. The three companion papers test the framework of the present paper in a setting where we have full control on what the answer to various questions must be: Free scalar field theories on Minkowski space in arbitrary dimensions. Note, however, that the framework developed here is background independent. Instead of using resolution scales with respect to a background metric that are used in these companion papers one can abstract to partially ordered and directed label sets which in fact motivated this article as we have applications in Loop Quantum Gravity in mind [49-52]. The language developed here could also be applied directly to the asymptotic safety approach to quantum gravity [53-56], a task to which we want to come back in the future.

In the first companion paper [57] we analytically derive the Hamiltonian renormalisation flow for the 1+1-dimensional, massive Klein Gordon model and compute its fixed points. The restriction to $1+1$ dimensions helps illustrating the basic mechanisms without exceedingly cluttering the formulae. We compare the direct and path integral induced flow and find a surprise, as could be anticipated from the general framework of the present paper: The path integral induced and direct Hamiltonian flow drastically differ from each other and only the coarse grained versions of the direct Hamiltonian flow qualify as the matrix elements of the continuum Hamiltonian between coarse grained vector states.

In the second companion paper [58] we investigate further properties of the renormalisation flow of the 1+1-dimensional model such as i. whether the flow in fact converges to the fixed point computed, ii. the decay properties of the correlations of the fixed point Laplacian with respect to distance between lattice points and iii. universality properties of the flow (dependence on the coarse graining map and initial data).

Finally, in the third companion paper [59] we consider D+1 dimensional free scalar quantum fields. It turns out that the analytical methods used for the $1+1$ case can be directly transferred due to a certain factorisation property. The flow has a fixed point which agrees with the Euclidian (or rotationally) invariant continuum theory. This enables us to study the question whether there is a criterion that enables one to decide at finite resolution, i.e. without taking the continuum limit, i.e. on a finite lattice, whether the flow converges to a Euclidian (or rotationally) invariant theory. We define such a criterion using irrational Euler angle techniques.

\section{Bijection between OS measure and OS data}

We begin in the first subsection with some preliminaries that explain the class of theories considered and prepare the language and assumptions necessary in order that a suitable bijection between path integral and Hamiltonian formulation can be stated. In the next subsection of this section we construct the OS measure from OS data based on the idea of the Wiener measure or the FeynmanKac formula, see e.g. [20] and references therein. Then we perform the more standard converse procedure which we include here only for the sake of completeness and in order to introduce a 
uniform notation, see e.g. [1] and references therein. In the third section we show, under the made restrictions, that both constructions are inverses of each other. We believe that our presentation of partly well known material will be useful to a mixed audience, in particular, because we employ a background independent and generally theory independent language.

\subsection{Preliminaries}

Let us first explain the general setting:

We consider a general theory based on a classical Lagrangian (or Hamiltonian) which is not explicitly coordinate dependent. We leave the field content open, but assume that diffeomorphism type gauge symmetries have been taken care of by suitable gauge fixings. In particular, even in generally covariant theories, after suitable gauge fixing of the timelike diffeomorphisms, there is a preferred notion of time, see e.g. [60-63] and references therein.

In more detail, as we are interested in situations with a well posed classical initial value formulation, the underlying spacetimes under consideration are globally hyperbolic so that points in the spacetime manifold $M$ can be denoted by $(t, x)$ where time $t$ takes real values and $x$ is a point in some fixed submanifold $\sigma$ of $M$ of codimension one. It follows from our assumptions that all time zero fields, collectively denoted by $\phi(x)$ and their canonical momenta, collectively denoted by $\pi(x)$, are defined with respect to a physical coordinate system. We further assume that $(\phi, \pi)$ have standard canonical brackets which is not easy to achieve due to the gauge fixing conditions which typically add corrections to the canonical (Dirac) bracket, but is possible for suitable reference matter fields, see e.g. [63].

On the other hand, usual Yang-Mills type gauge symmetries need not have to be gauge fixed. Instead, we assume that in this case one employs gauge covariant versions of the usual Weyl elements $[64,65] w(f, g):=\exp (i[\phi(f)+\pi(g)])$ that one uses in scalar field theories. For Bose fields, $(\phi, \pi)$ and $(g, f)$ are geometrically both a pair consisting of a spatial scalar density of weight zero and one respectively such that the pairing $\phi(f)=\int_{\sigma} d^{D} x f \phi$ is invariant under active spatial diffeomorphisms ( $f, F$ are real valued). Thus, for instance for a non-Abelian Yang-Mills theory or General Relativity when formulated in terms of connection variables [66-68] one will utilise Wilson loop type holonomy variables for the spatial connection $\phi$ and exponentiated non-Abelian electric flux variables for the conjugate electric field $\pi$. We will treat the case of Fermi fields in a future publication.

In order to be theory independent, we will talk about the corresponding generalised Weyl elements $w(f, g), w(f):=w(f, g=0)$ for the canonical formulation in what follows as well as the canonical Hamiltonian $H=H(\phi, \pi)$ which is supposed to be a positive functional of $\phi, \pi$ without explicit coordinate dependence, i.e. the system is conservative.

We are interested in (non-degenerate) representations of the ${ }^{1} *$-Algebra $\mathfrak{A}$ generated by the Weyl elements that supports the Hamiltonian $H$ as a self-adjoint operator. In the case of an interacting quantum field theory this will generically require ultra-violet and infra-red regularisations. With such regulators in place, let $t \mapsto \alpha_{t}$ denote the one parameter group of (outer) automorphisms of $\mathfrak{A}$ corresponding to the classical Hamiltonian flow of $H$. As every (automatically non-degenerate as $\mathfrak{A}$ is unital) representation is a direct sum of cyclic ones [65] and every such representation $(\rho, \mathcal{H}, \Omega)$ is generated by a positive linear functional $\omega$ by the GNS construction [71] we are interested in invariant states $\omega \circ \alpha_{t}=\omega$. Namely, in this situation we have a representation by inner automorphisms

$$
\rho \circ \alpha_{t}=U(t) \circ \rho \circ U(t)^{-1}, \quad U(t)=: \exp (-i H t)
$$

where the one parameter group $t \mapsto U(t)$ of unitarities is generated by $H$ according to Stone's theorem if $t \mapsto U(t)$ is strongly continuous, as we will assume. Note that $\Omega$ is a zero eigenvector of

\footnotetext{
${ }^{1}$ Whether or not $\mathfrak{A}$ can be equipped with a unique $C^{*}$-algebra structure on purely algebraic grounds such as in the case of symplectic vector spaces [65] will not be investigated here, see e.g. [69,70] for the case of Yang-Mills fields.
} 
$H$, i.e. a vacuum vector. For convenience we will assume that $H$ is bounded from below (without loss of generality by zero) just as the classical object.

As already mentioned we will treat Fermi fields separately in a future publication and focus on Bose fields for the rest of this work. For bosonic theories of interest (the Hamiltonian is quadratic in the momenta), $\rho(w[f, t]):=U(t) \rho(w[f]) U(t)^{-1}=\rho\left(\alpha_{t}(w[f])\right)$ contains sufficient information about $\pi(g)$ as well (take the time derivative at $t=0$ ) so that one can focus attention on formulating a quantum field theory in terms of the Heisenberg field operators $\rho(w[f, t])$ in what follows. Now it is in general expected that for generic representations $\rho$ of an interacting quantum field theory the sharp time objects $\rho(w[f, t])$ are no longer represented as operators but rather as operator valued distributions (with respect to the time variable) when we take the regulators away (and renormalise the fields) so that $\rho(w[f, t])$ necessarily also must be smeared in the time coordinate in order to obtain a well defined operator. For instance, this may be viewed as a consequence of Haag's theorem [71] for quantum fields on Minkowski space in the context of the Wightman axiomatic framework. Hence, the formulation of a quantum field theory based on the $\rho(w[f, t])$ of which we make extensive use throughout this series of papers appears to be of little relevance for the most interesting models, even if the triple of Osterwalder-Schrader data $(\mathcal{H}, \Omega, H)$ survives the regulator limit as we assume throughout this series.

However, while this is generically certainly true for the $\rho(w[f, t])$ as defined starting from the classical time zero field $\phi$, under one rather mild technical assumption there exists a reformulation in terms of which sharp time field operators do make sense, albeit for generically different fields. Namely, we will assume throughout the whole paper, that the final Hilbert space $\mathcal{H}$ that one ends up with admits an Abelian $C^{*}$-subalgebra $\mathfrak{B}$ of the $C^{\star}$-algebra $\mathcal{B}(\mathcal{H})$ of bounded operators on $\mathcal{H}$ for which the final vacuum vector state $\Omega$ that one ends up with is cyclic. This assumption is not very restrictive, it is easy to see that it holds for instance when $\mathcal{H}$ is separable but also in many non-separable cases. In fact, in our renormalisation flow application, the Hilbert spaces that we encounter at finite resolution are all separable, hence our reformulation that we are about to sketch certainly is valid in the applications that we have in mind in this series of works.

Let $\Delta(\mathfrak{B})$ be the Gel'fand spectrum of $\mathfrak{B}[72]$. Then we will call the points $\phi^{\prime}$ of $\Delta(\mathfrak{B})$ quantum fields at sharp time zero. Of course, in general the relation between the quantum sharp time zero field $\phi^{\prime}$ and the classical sharp time zero field $\phi$ will be rather involved and depends on the choice of $\mathfrak{B}$. Taking into account of what was just said, we expect that $\phi^{\prime}$ is related to a certain time average of $\phi$. Indeed, in the course of this series of papers we will see an instance of this effect when we renormalise the free Klein-Gordon field.

Using the Gel'fand isomorphism [72] we may think of $\mathfrak{B}$ as $C(\Delta(\mathfrak{B}))$, the continuous functions on the spectrum. Since the spectrum carries a compact Hausdorff topology, the positive linear and normalised expectation value functional $\langle\Omega, \Omega\rangle_{\mathcal{H}}$ on $\mathfrak{B}$ gives rise to a probability measure $\nu$ on $\Delta(\mathfrak{B})$ by the Riesz-Markov theorem [73]. It follows that $\mathcal{H} \cong L_{2}(\Delta(\mathfrak{B}), d \nu)$. If necessary, we can perform a unitary transformation such that the vacuum becomes the constant vector $\Omega^{\prime}=1$. This is possible if $\Omega$ is not vanishing $\nu$ almost everywhere (a.e.). The new representation is given by $\rho^{\prime}()=.\Omega^{-1} \rho(.) \Omega, \psi^{\prime}=\Omega^{-1} \psi, \nu^{\prime}=|\Omega|^{2} \nu$ where we understand $\Omega$ as a multiplication operator.

We consider a new generating set of $\mathfrak{B}$ consisting of bounded functions $w^{\prime}\left[f^{\prime}\right]$ on $\Delta(\mathfrak{B})$ to which we refer as new Abelian Weyl elements. Here $f^{\prime}$ belongs to a certain label set $L^{\prime}$ of smearing functions on $\sigma$ (for instance functions of rapid decrease for free scalar fields) and each $b \in \mathfrak{B}$ is supposed to be in the finite linear span of the $\rho\left(w^{\prime}\left[f^{\prime}\right]\right)$. For this to be consistent we require Weyl type relations among the $w^{\prime}\left[f^{\prime}\right]$ which allow us to express $\left(w^{\prime}\left[f^{\prime}\right]\right)^{*}$ and $w^{\prime}\left[f_{1}^{\prime}\right] \cdot w^{\prime}\left[f_{2}^{\prime}\right]$ as a finite linear combination of the $w^{\prime}\left[f^{\prime}\right]$ again. Specifically we could have relations of the form

$$
\left(w^{\prime}\left[f^{\prime}\right]\right)^{*}=: w^{\prime}\left[\left(f^{\prime}\right)^{*}\right], w^{\prime}\left[f_{1}^{\prime}\right] w^{\prime}\left[f_{2}^{\prime}\right]=: \sum_{\alpha=1}^{N} z_{\alpha} w^{\prime}\left[f_{\alpha}^{\prime}\left(f_{1}^{\prime}, f_{2}^{\prime}\right)\right]
$$

where $N<\infty, z_{\alpha} \in \mathbb{C}$ are independent of $f_{1}^{\prime}, f_{2}^{\prime}$ and the new smearing function $f_{\alpha}^{\prime}$ also is built from $f_{1}^{\prime}, f_{2}^{\prime}$ in the same way for any $f_{1}^{\prime}, f_{2}^{\prime}$. Also, $\left(f^{\prime}\right)^{*}$ is not the complex conjugate of $f^{\prime}$ (which 
is typically real valued) but rather determined by the fact that $\left(w^{\prime}\left[f^{\prime}\right]\right)^{*}$ is the adjoint of $w^{\prime}\left[f^{\prime}\right]$ and coincides with the complex conjugate $\overline{w^{\prime}\left[f^{\prime}\right]}$ in our reformulated representation in which $w^{\prime}\left[f^{\prime}\right]$ acts by multiplication. For a free scalar field of course $\left(f^{\prime}\right)^{*}=-f^{\prime}, N=1, z_{1}=1, f_{\alpha=1}^{\prime}\left(f_{1}^{\prime}, f_{2}^{\prime}\right)=f_{1}^{\prime}+f_{2}^{\prime}$. For non-Abelian gauge theories formulated in terms of Wilson loop variables, the relations (2.2) are known as Mandelstam identities [6].

The Weyl elements at sharp time $w^{\prime}\left[f^{\prime}, t\right]=U(t) \rho\left(w^{\prime}\left[f^{\prime}\right]\right) U(t)^{-1}$ as a product of three bounded operators now make perfect sense as operators on $\mathcal{H}$. Moreover, the vacuum is cyclic for the $\rho\left(w^{\prime}\left[f^{\prime}\right]\right)$ In what follows we will drop the primes and the representation label $\rho$ in order to simplify the formulae.

\subsection{Constructing the OS measure from OS data}

We consider the set of generating functionals of the Wightman functions (as $N$ varies)

$$
\begin{aligned}
W\left(\left(f_{N}, t_{N}\right), . .,\left(f_{1}, t_{1}\right)\right) & :=\left\langle\Omega, w\left(f_{N}, t_{N}\right) . . w\left(f_{1}, t_{1}\right) \Omega\right\rangle_{\mathcal{H}} \\
& =\left\langle\Omega, w\left(f_{N}\right) U\left(t_{N}-t_{N-1}\right)^{-1} . . U\left(t_{2}-t_{1}\right)^{-1} w\left(f_{1}\right) \Omega\right\rangle_{\mathcal{H}}
\end{aligned}
$$

If the labels $f$ correspond to semaring functions, then formally the actual Wightman functions are derived from (2.3) by taking the functional derivatives with respect to the sharp time smearing functions $f_{1}, . ., f_{N}$ and then setting them to zero.

By definition, the Schwinger functions arise from the Wightman functions by analytic continuation in time $t \mapsto i \beta, \beta \in \mathbb{R}$, provided, the continued times are ordered $\beta_{N}>\beta_{N-1}>. .>\beta_{1}$ so that the unitary time evolution operator becomes a self-adjoint contraction operator

$$
\left.U\left(t_{k}-t_{k-1}\right)^{-1}=\exp \left(i\left(t_{k}-t_{k-1}\right) H\right) \mapsto \exp \left(-\left(\beta_{k}-\beta_{k-1}\right) H\right)\right)
$$

which is bounded in operator norm by unity thanks to the positivity of $H$. Accordingly, we obtain the $\mathrm{N}$-point Schwinger function generator without domain questions

$$
S\left(\left(f_{N}, \beta_{N}\right), . .,\left(f_{1}, \beta_{1}\right)\right):=\left\langle\Omega, w\left(f_{N}\right) e^{-\left[\beta_{N}-\beta_{N-1}\right] H} . . e^{-\left[\beta_{2}-\beta_{1}\right] H} w\left(f_{1}\right) \Omega\right\rangle_{\mathcal{H}}
$$

To define a path integral measure $\mu$ from (2.5) we consider a spacetime field $\Phi$ taking values in some set $\Gamma$ to be specified (namely the support of $\mu$ ) and the cylindrical sharp time subsets of $\Gamma$ defined by

$$
\Gamma_{B_{1}, . ., B_{N}}^{\beta_{1}, . . \beta_{N}}=\left\{\Phi \in \Gamma ; \Phi\left(\beta_{k}\right) \in B_{k} ; k=1, . ., N\right\}
$$

where $\beta_{1}<. .<\beta_{N}$ are real numbers and $B_{k}$ are Borel sets with respect to the measure $\nu$ on $\Delta(\mathfrak{B})$ considered above. The set $\Gamma$ itself is also cylindrical and corresponds to the case $N=2, B_{1}=B_{2}=$ $\Delta(\mathfrak{B})$ with any $\beta_{1}<\beta_{2}$ (no restriction). Then we assign to (2.6) the "heat kernel" measure (heat kernel with respect to $H$ )

$$
\mu\left(\Gamma_{B_{1}, \ldots, B_{N}}^{\beta_{1}, \ldots, \beta_{N}}\right):=\left\langle\Omega, \chi_{B_{N}} e^{-\left[\beta_{N}-\beta_{N-1}\right] H} . . e^{-\left[\beta_{2}-\beta_{1}\right] H} \chi_{B_{1}} \Omega\right\rangle
$$

where $\chi_{B}$ is the operator that multiplies by $\chi_{B}(\phi), \phi \in \Delta(\mathfrak{B})$ on the Hilbert space $L_{2}(\Delta(\mathfrak{B}), d \nu)$. Note that $\mu(\Gamma)=1$ due to $H \Omega=0$ and $\chi_{\Delta(\mathfrak{B})}=1_{\mathcal{H}}$. The latter fact is responsible for the fact that our definition is cylindrically consistent (adding time slots with $1_{\mathcal{H}}$ insertions does not affect the answer). Apart from this, it is however unclear whether the numbers (2.7), although finite, are positive. If they are, they define the Wiener measure with respect to $H$.

For a general theory it is a very difficult problem to decide whether (2.7) is positive. In the literature, this property is known as Nelson-Symanzik positivity [4,74]. While in general, (2.7) need not be positive (cf. [7] for a counter example), a more succinct criterion for its positivity is available. Under the assumption of a vacuum $\Omega$ cyclic for $\mathfrak{B}$, the positivity can be reduced to the following positivity condition on $e^{-\beta H}$ : For any $F, G$ bounded, positive, measurable functions on the $\mathrm{N}$-fold and M-fold copies of the ranges of the functions $w[f]$ (e.g. $\mathbb{C}^{N}$ and $\mathbb{C}^{M}$ respectively for scalar fields) and test functions $f_{1}, \ldots, f_{N}, g_{1}, \ldots, g_{M}$, it suffices to show that

$$
\left\langle F\left(w\left(f_{1}\right), \ldots, w\left(f_{N}\right)\right), e^{-\beta H} G\left(w\left(g_{1}\right), \ldots, w\left(g_{M}\right)\right)\right\rangle \geq 0 .
$$


For completeness, we sketch the proof in appendix B.

In what follows we assume that the Wiener measure $\mu$ defined cylindrically in (2.7) exists. Then one can define the integral of simple functions (linear combinations of characteristic functions of cylindrical sets as in (2.6)) and approximate the integral of more complicated functions by simple functions, see e.g. [73] for the measure theoretic details of this construction process. For (2.6) we have

$$
\chi_{\Gamma_{\left\{B_{k}\right\}}^{\left\{\beta_{k}\right\}}}[\Phi]=\prod_{k=1}^{N} \chi_{B_{k}}\left(\Phi\left(\beta_{k}\right)\right)
$$

and one sets

$$
\mu\left(\chi_{\Gamma_{\left\{B_{k}\right\}}^{\left\{\beta_{k}\right\}}}[\Phi]\right):=\mu\left(\Gamma_{\left\{B_{k}\right\}}^{\left\{\beta_{k}\right\}}\right)=\mu\left(\prod_{k=1}^{N} \chi_{B_{k}}\left(\Phi\left(\beta_{k}\right)\right)\right.
$$

In view of (2.5) we are in particular interested in functions $G[\Phi]$ which depend on $\Phi$ only through $\Phi\left(\beta_{k}\right), k=1, . ., N$ for a finite number of times. These can be approximated by a sequence $G_{n}$ of linear combinations of step functions as in (2.9) for fixed $\beta_{k}$ but each $G_{n}$ involves many $B_{k}$. Concretely, one partitions $\Delta(\mathfrak{B})^{N}$ into mutually disjoint cells of the form $B_{1} \times \ldots \times B_{N}$, picks a point in such a cell and approximates $G$ by the function that assumes the value of $G$ at this point throughout the whole cell. It is clear such an approximant is an (infinite) linear combination of functions (2.9) where the sum is over all cells of the partition. The integral $\mu(G)$, if it exists, is then defined by taking the limit of the integrals of the sequence ${ }^{2}$.

Let now

$$
W[F]_{F=\delta_{\beta_{k}} f_{k}}:=w\left[f_{k}\right]_{\phi \rightarrow \Phi\left(\beta_{k}\right)}
$$

that is, $W\left[\delta_{\beta_{k}} f_{k}\right]$ is exactly built as the configuration field Weyl element $w\left[f_{k}\right]$, just that the time zero operator field $\phi(x), x \in \sigma$ is replaced by the history integration field $\Phi\left(\beta_{k}, x\right)$ at time $\beta_{k}$. Then due to the fundamental relation (2.7) and what we have just said about the approximation by simple functions we can define

$$
\mu\left(\prod_{k=1}^{N} W\left(\delta_{\beta_{k}} f_{k}\right)\right)=\left\langle\Omega, w\left(f_{N}\right) e^{-\left(\beta_{N}-\beta_{N-1}\right) H} w\left(f_{N-1}\right) \ldots e^{-\left(\beta_{2}-\beta_{1}\right) H} w\left(f_{1}\right) \Omega\right\rangle=S\left(\left\{f_{k}, \beta_{k}\right\}\right)
$$

Since the history field is just an integration variable, the Schwinger function can be extended, using the measure $\mu$, to a function symmetric in all time variables in contrast to the Wightman function of course. That is why the sequence of the product occurring on the left handside of (2.12) is irrelevant.

Definition (2.12) defines $\mu$ on the history smearing fields of compact and sharp time support

$$
F:=\left(\delta_{\beta_{k}} f_{k}\right)_{k=1}^{N}, \quad W[F]:=\prod_{k} W\left[\delta_{\beta_{k}} f_{k}\right]
$$

Note that we simply define $W[F]$ this way. For scalar fields, we may independently define $W[F]:=$ $\exp \left(i \int d^{D+1} x F(x) \Phi(x)\right)$ which is consistent with (2.13) but for more general theories such as non-Abelian gauge theories for which $w[f]$ may for instance mean a Wilson loop variable $w[f]:=$ $\operatorname{Tr}\left(\operatorname{Hol}_{f}(\phi)\right)$ where $\phi$ is a connection, $f$ the distributional smearing function associated with a loop in $\sigma$ and $\mathrm{Hol}$ the holonomy map, the product of these Wilson loop variables cannot be expressed in terms of a time integral.

Remark: Equation (2.12) holds for any $N$ and this is sufficient in order to reconstruct the Schwinger functions. One sometimes wishes to take the limit $N \rightarrow \infty$ in order to obtain a generating functional. To

\footnotetext{
${ }^{2}$ More in detail one does this first for positive functions, shows that there exists a sequence of simple functions smaller than $G$ pointwise a.e. wrt $\mu$ and then takes the supremum of the integral over all simple functions that are smaller than $G$ a.e. wrt $\mu$. The integral for complex valued functions is defined by decomposing real and imaginary part in positive and negative contributions.
} 
that end we consider a single function $F$ on $\mathbb{R} \times \sigma$ of compact time support in $[-\beta, \beta]$ and let $\beta_{k}=\beta \frac{k}{N}, \beta>0, k=-N,-N+1, . ., N$. Then we set

$$
f_{k}:=\int_{\beta_{k}-\beta /(2 N)}^{\beta_{k}+\beta /(2 N)} d \beta^{\prime} F\left(\beta^{\prime}\right)
$$

to be the average value of the single function $F$ over an interval of width $\beta / N$ around the sharp time $\beta_{k}$. If we formally define

$$
W[F]:=\lim _{N \rightarrow \infty} \prod_{k=-N}^{N} W\left(\delta_{\beta_{k}} f_{k}\right)
$$

one can try to give meaning to the limit

$$
\mu(W[F])=\lim _{N \rightarrow \infty}\left\langle\Omega, w\left(f_{N}\right) e^{-\frac{\beta}{N} H} w\left(f_{N-1}\right) . . e^{-\frac{\beta}{N} H} w\left(f_{-N}\right) \Omega\right\rangle
$$

For a scalar field (2.15) converges to

$$
W[F, \Phi]=\exp (i \Phi[F]), \quad \Phi[F]=\int_{\mathbb{R} \times \sigma} d \beta d^{D} x \Phi(\beta, x) F(\beta, x)
$$

and $\mu(W[F])$, when it exists, is the generating functional of the Schwinger functions of a scalar field which one obtains by functional derivation with respect to $F$. For a non-Abelian gauge theory such a simple formula is not available. That is why in this more general case we refer to (2.12) as the Schwinger function. Notice that we recover (2.12) from (2.16) for the sharp time smearing functions of the form

$$
F(\beta)=\sum_{k=1}^{N} f_{k} \delta\left(\beta, \beta_{k}\right)
$$

so that (2.12) can be considered as the more fundamental object while (2.16) is a derived concept which is useful when it exists and then reproduces (2.12). It transpires that the measure is determined by $F$ with finite, discrete time support and we will deal only with that case in what follows as it applies also to quantum fields more general than scalar ones.

We claim that $\mu$ so constructed is automatically time reflection invariant, time translation invariant and reflection positive. To define these notions, let $\mathcal{H}^{\prime}:=L_{2}(\Gamma, d \mu)$ be the history Hilbert space defined as the closed linear span of the functions $W[F]$ equipped with the scalar product

$$
\left\langle W[F], W\left[F^{\prime}\right]\right\rangle_{\mathcal{H}^{\prime}}:=\mu\left(\overline{W[F]} W\left[F^{\prime}\right]\right)
$$

Notice that the Weyl relations (2.2) are inherited by the functions $W[F]$ since the $W[F]$ are products of functions of the form $w[f]$ with $\phi$ replaced by $\Phi(\beta)$ for some $\beta$. That is, whenever $W[F], W\left[F^{\prime}\right]$ have coinciding points of time, the rules (2.2) apply in $W[F] W\left[F^{\prime}\right]$ otherwise we just prolong the list of points of time. In particular, if $F, F^{\prime}$ have positive time support then we have $W[F] W\left[F^{\prime}\right]=W[\tilde{F}]$ for certain $\tilde{F}$ which still has positive time support. For a scalar field for instance $\overline{W[F]} W\left[F^{\prime}\right]=$ $W\left[F^{\prime}-F\right]$.

We define time reflection on the smearing functions and the history Hilbert space densely by

$$
(\theta F)(\beta):=F(-\beta), \quad R W[F]:=W[\theta F]
$$

We begin by showing that the measure $\mu$ is automatically reflection invariant. For $F(t)=\left(\delta_{\beta_{k}}(t) f_{k}\right)_{k=1}^{N}$ with $\beta_{1}<. .<\beta_{N}$ we have $(\theta \cdot F)(t)=\left(\delta_{-\beta_{k}}(t) f_{k}\right)_{k=1}^{N}$ whence due to $-\beta_{N}<. .<-\beta_{1}$ by definition 
of $\mu$

$$
\begin{aligned}
\mu(R \cdot W[F]) & =\left\langle\Omega, w\left[f_{1}\right] e^{-\left(-\beta_{1}-\left[-\beta_{2}\right]\right) H} w\left[f_{2}\right] e^{-\left(-\beta_{2}-\left[-\beta_{3}\right]\right) H} . . e^{-\left(\beta_{N-1}-\left[-\beta_{N}\right]\right) H} w\left[f_{N}\right] \Omega\right\rangle \\
& =\left\langle\Omega, w\left[f_{1}\right] e^{-\left(\beta_{2}-\beta_{1}\right) H} w\left[f_{2}\right] e^{-\left(\beta_{3}-\beta_{2}\right) H} . . e^{-\left(\beta_{N}-\beta_{N-1}\right) H} w\left[f_{N}\right] \Omega\right\rangle \\
& =\left\langle w\left[f_{N}\right]^{*} e^{-\left(\beta_{N}-\beta_{N-1}\right) H} . . e^{-\left(\beta_{2}-\beta_{1}\right) H} w\left[f_{1}\right]^{*} \Omega, \Omega\right\rangle \\
& =\overline{\left\langle\Omega, w\left[f_{N}^{*}\right] e^{-\left(\beta_{N}-\beta_{N-1}\right) H} . . e^{-\left(\beta_{2}-\beta_{1}\right) H} w\left[f_{1}^{*}\right] \Omega\right\rangle} \\
& =\overline{\mu\left(W\left[F^{*}\right]\right)}=\int_{\Gamma} d \mu(\Phi) \overline{W\left[F^{*}\right]} \\
& =\mu\left(W\left[F^{*}\right]^{*}\right)=\mu(W[F])
\end{aligned}
$$

where positivity of the measure was used as well as involutivity of *. It follows that $R$ is a unitary operator on $\mathcal{H}^{\prime}$ due to $R^{2}=1_{\mathcal{H}^{\prime}}$.

We consider the subspace $V$ of $\mathcal{H}^{\prime}$ consisting of the finite linear span of the $W[F]$ where $F$ has positive finite and discrete time support in $(0, \infty)$. Then $\mu$ is said to be reflection positive iff

$$
\langle\Psi, R \Psi\rangle_{\mathcal{H}^{\prime}} \geq 0 \quad \forall \Psi \in V
$$

We consider an arbitrary element of $V$ which can be written as

$$
\Psi=\sum_{I=1}^{M} z_{I} W\left[F^{I}\right], \quad \mathrm{T}-\operatorname{supp}\left(F^{I}\right) \subset(0, \infty)
$$

and work out condition (2.22). The crucial observation is that if $\mathrm{T}-\operatorname{supp}(F) \subset(0, \infty)$ then $\mathrm{T}$ $\operatorname{supp}(\theta F) \subset(-\infty, 0)$. Suppose that $F^{I}=\sum_{k=1}^{N^{I}} \delta_{\beta_{k}^{I}} f_{k}^{I}, 0<\beta_{1}^{I}<. .<\beta_{N^{I}}^{I}$ then similarly as above

$$
\begin{aligned}
& \langle\Psi, R \Psi\rangle=\sum_{I, J} \bar{z}_{I} z_{J} \mu(\overline{W[F]} W[\theta \cdot F]) \\
& =\sum_{I, J} \bar{z}_{I} z_{J} \mu\left(W\left[F^{*}\right] W[\theta \cdot F]\right) \\
& =\sum_{I, J} \bar{z}_{I} z_{J}\left\langle\Omega, w\left[\left(f_{N^{I}}^{I}\right)^{*}\right] e^{-\left(\beta_{N^{I}}^{I}-\beta_{N^{I}-1}^{I}\right) H} . . e^{-\left(\beta_{2}^{I}-\beta_{1}^{I}\right) H} w\left[\left(f_{1}^{I}\right)^{*}\right] \times\right. \\
& \left.\times e^{-\left(\beta_{1}^{I}-\left[-\beta_{1}^{J}\right]\right) H} w\left[f_{1}^{J}\right] e^{-\left(\beta_{2}^{J}-\beta_{1}^{J}\right) H} . . e^{-\left(\beta_{N^{J}}^{J}-\beta_{N^{J}-1}^{J}\right) H} w\left[f_{N^{J}}^{J}\right] \Omega\right\rangle \\
& =\sum_{I, J} \bar{z}_{I} z_{J}\left\langle e^{-\beta_{1}^{I} H} w\left[f_{1}^{I}\right] e^{-\left(\beta_{2}^{I}-\beta_{1}^{I}\right) H} . . e^{-\left(\beta_{N^{I}}^{I}-\beta_{N^{I}-1}^{I}\right) H} w\left[f_{N^{I}}^{I}\right] \Omega\right. \text {, } \\
& \left.e^{-\beta_{1}^{J} H} w\left[f_{1}^{J}\right] e^{-\left(\beta_{2}^{J}-\beta_{1}^{J}\right) H} . . e^{-\left(\beta_{N}^{J}{ }^{J}-\beta_{N}^{J}{ }_{-1}\right) H} w\left[f_{N^{J}}^{J}\right] \Omega\right\rangle \\
& =\left\|\sum_{I} z_{I} e^{-\beta_{1}^{I} H} w\left[f_{1}^{I}\right] e^{-\left(\beta_{2}^{I}-\beta_{1}^{I}\right) H} . . e^{-\left(\beta_{N^{I}}^{I}-\beta_{N^{I}-1}^{I}\right) H} w\left[f_{N^{I}}^{I}\right] \Omega\right\|^{2}
\end{aligned}
$$

which is manifestly non-negative.

Finally, we show that $\mu$ is trivially time translation invariant. We define densely on $\mathcal{H}^{\prime}$

$$
\left(T_{s} F\right)\left(\beta^{\prime}\right):=F\left(\beta^{\prime}-s\right), \mathcal{U}(s) W[F]:=W\left[T_{s} F\right]
$$

Thus for $F=\left(\delta_{\beta_{k}} f_{k}\right)_{k=1}^{N}, \beta_{1}<. .<\beta_{k}$ we have $T_{s} \cdot F=\left(\delta_{\beta_{k}+s} f_{k}\right)_{k=1}^{N}$ hence with $\beta_{k}^{\prime}=\beta_{k}+s$ we still have $\beta_{1}^{\prime}<. .<\beta_{N}^{\prime}$ so that

$$
\mu(\mathcal{U}(s) W[F])=\left\langle\Omega, w\left[f_{N}\right] e^{-\left(\beta_{N}^{\prime}-\beta_{N-1}^{\prime}\right) H} . . e^{-\left(\beta_{2}^{\prime}-\beta_{1}^{\prime}\right) H} w\left[f_{1}\right] \Omega\right\rangle=\mu(W[F])
$$

since $\beta_{k}^{\prime}-\beta_{l}^{\prime}=\beta_{k}-\beta_{l}$. Note that $s \mapsto \mathcal{U}(s)$ is in particular a one-parameter unitary group on $\mathcal{H}^{\prime}$. Now the strong continuity of the one parameter unitary group $t \mapsto U(t)=e^{-i t H}$ on $\mathcal{H}$, equivalent to the existence of the self-adjoint generator $H$ by Stone's theorem, implies strong continuity 
of the one-parameter semi-group $\beta \mapsto e^{-\beta H}$ with $\beta>0$ on $\mathcal{H}$. This in turn implies the strong continuity of the unitary group $\mathcal{U}(s)$. As we will see in the next section, the bi-linear form (2.22) is positive but not definite and thus has a null space which leads to corresponding equivalence classes $[\Psi], \Psi \in V$. The space $V$ is only invariant under $\mathcal{U}(s)$ for $s \geq 0$ and thus gives rise to a semigroup $K^{\prime}(s)[\Psi]:=[\mathcal{U}(s) \Psi]$ (see the next section for proving independence of the representative). It follows that the semigroup $s \mapsto K^{\prime}(s)$ is also strongly continuous, a property that maybe called "reflection continuity" and which implies the existence of a generator of the semigroup which gives rise to a Hamiltonian defined within the measure theoretic framework, see the next subsection.

In conclusion, we have shown that the Hamiltonian framework, under the assumption of positivity of (2.7) and under the assumptions made in the preliminaries, always gives rise to a measure on an appropriate history field space $\Gamma$ which is time translation and time reflection invariant as well as reflection positive and continuous.

We will not require the remaining Osterwalder-Schrader axioms, that is, Euclidian invariance of the measure, analyticity of the generating functional in the smearing fields $F$, regularity as an estimate of the generating functional in terms of $L_{p}$ norms of $F$ and clustering ${ }^{3}$ of the measure with respect to time translations. Euclidian invariance maybe too strong to ask for our theory under investigation (e.g. General Relativity), analyticity maybe too strong to ask if the state $\omega$ is not regular for $\mathfrak{B}$ (i.e. not strongly continuous with respect to $F$ in $W[F]$ ) in which case also the regularity OS axiom will fail to hold and finally ergodicity, which is equivalent to the uniqueness of the vacuum of $H$ cannot be verified here unless we put this in from the outset into the canonical theory, which we do not want to do because in more general theories, the vacuum sector maybe degenerate (phase transitions).

\subsection{Constructing the OS data form the OS measure}

The converse statement is standard, we include it here only for completeness, to have a unified notation and in order to draw attention to the fact that only reflection invariance and reflection positivity as well as time translation invariance and continuity are needed in order to construct the OS data. Otherwise we will be brief, see [1] for all the details in a Minkowski space context and [10] for the context in a generally covariant theory.

We assume to be given a time reflection and time translation invariant as well as reflection positive and continuous measure on the history field space $\Gamma$. By definition of reflection positivity, the bi-linear form on $V$, which is the space of the finite linear combinations of the $W[F]$ with $F$ of strictly positive time support, given by

$$
\left(\Psi, \Psi^{\prime}\right)_{V}:=\left\langle\Psi, R \Psi^{\prime}\right\rangle_{\mathcal{H}^{\prime}}
$$

is positive but not necessarily positive definite on $V$. However, due to reflection invariance, in particular unitarity of the time reflection operator $R$, it is actually sesqui-linear

$$
\overline{\left(\Psi, \Psi^{\prime}\right)_{V}}=\left\langle R \Psi^{\prime}, \Psi\right\rangle_{\mathcal{H}^{\prime}}=\left\langle R^{2} \Psi^{\prime}, R \Psi\right\rangle_{\mathcal{H}^{\prime}}=\left(\Psi^{\prime}, \Psi\right)_{V}
$$

We consider the null space

$$
\mathcal{N}:=\{\Psi \in V ;(\Psi, \Psi)=0\}
$$

and define the canonical Hilbert space $\mathcal{H}$ as the completion of the equivalence classes $[\Psi]:=\{\Psi+$ $\left.\Psi_{0}, \Psi_{0} \in \mathcal{N}\right\}$ with $\Psi \in V$ with respect to the scalar product

$$
\left\langle[\Psi],\left[\Psi^{\prime}\right]\right\rangle_{\mathcal{H}}:=\left(\Psi, \Psi^{\prime}\right)_{V}
$$

\footnotetext{
${ }^{3}$ Alternatively: ergodicity of the measure with respect to time translations, that is, time average of $\Psi \in L_{1}(\mu)$ and ensemble average $\mu(\psi)$ coincide $\mu$ a.e.
} 
This definition is independent of the representative by virtue of the Cauchy-Schwarz inequality which applies to positive semi-definite sesquilinear forms. Note that $\mathrm{T}-\operatorname{supp}\left(T_{s} F\right)=\mathrm{T}-\operatorname{supp}(F)+s$, thus the unitary operator densely defined on $\mathcal{H}^{\prime}$ by $\mathcal{U}(s) W[F]=W\left[T_{s} F\right]$ does not map $V$ onto $V$ unless $s>0$. Thus, on $V$ we may still define a one parameter family $s \mapsto \mathcal{U}(s)$, but it is a semi-group rather than a group because the inverses are not defined. We want to define for $\Psi \in V$

$$
K(s)[\Psi]:=[\mathcal{U}(s) \Psi]
$$

but must show that the definition is independent of the representative, i.e. that $\mathcal{U}(s) \mathcal{N} \subset \mathcal{N}$. We have

$$
\theta \circ T_{s} \cdot F(\beta)=\left(T_{s} \cdot F\right)(-\beta)=F(-\beta-s)=F(-(\beta+s))=(\theta \cdot F)(\beta+s)=T_{-s} \circ \theta \cdot F(\beta)
$$

whence

$$
R \mathcal{U}(s) W[F]=W\left[\theta \circ T_{s} F\right]=\mathcal{U}(-s) R W[F]
$$

We therefore have, using unitarity of $\mathcal{U}(s)$ on $\mathcal{H}^{\prime}$, for $\Psi \in V, \Psi_{0} \in \mathcal{N}$

$$
\begin{aligned}
\left|\left(\Psi, \mathcal{U}(s) \Psi_{0}\right)\right| & =\left|\left\langle\Psi, R \mathcal{U}(s) \Psi_{0}\right\rangle_{\mathcal{H}^{\prime}}\right|=\left|\left\langle\Psi, \mathcal{U}(-s) R \Psi_{0}\right)\right\rangle_{\mathcal{H}^{\prime}} \mid= \\
& \left.=\left|\left\langle\mathcal{U}(s) \Psi, R \Psi_{0}\right)\right\rangle_{\mathcal{H}^{\prime}}|=|\left(\mathcal{U}(s) \Psi, \Psi_{0}\right)\right\rangle_{V}|\leq| \mid \mathcal{U}(s) \Psi\left\|_{V} \quad\right\| \Psi_{0} \|_{V}=0
\end{aligned}
$$

The same calculation as in (2.34) demonstrates that $K(s)$ is symmetric on $\mathcal{H}$ and positive since

$$
\langle[\Psi], K(s)[\Psi]\rangle_{\mathcal{H}}=\left\langle[\Psi],\left[\mathcal{U}(s / 2)^{2} \Psi\right]\right\rangle_{\mathcal{H}}=\left\langle[\Psi], K(s / 2)^{2}[\Psi]\right\rangle_{\mathcal{H}}=\|K(s / 2)[\Psi]\|_{\mathcal{H}}^{2}
$$

We show that $K(s)$ is a contraction. Consider any $0 \neq[\Psi] \in \mathcal{H}$ (in particular $0 \neq \Psi \in \mathcal{H}^{\prime}$ ), then using the Cauchy-Schwarz inequality for $\mathcal{H}$ repeatedly

$$
\begin{aligned}
\|K(s)[\Psi]\|_{\mathcal{H}} & =\sqrt{\left\langle[\Psi], K(s)^{2}[\Psi]\right\rangle_{\mathcal{H}}} \\
& \leq\|[\Psi]\|^{1 / 2}\left[\|K(2 s)[\Psi]\|_{\mathcal{H}}\right]^{1 / 2} \\
& \leq\|[\Psi]\|^{\sum_{k=1}^{n} 2^{-k}}\left[\left\|K\left(2^{n} s\right)[\Psi]\right\|_{\mathcal{H}}\right]^{2^{-n}}
\end{aligned}
$$

Now for any $r>0$, using the unitarity of $R, \mathcal{U}(r)$ for $\mathcal{H}^{\prime}$ and its Cauchy-Schwarz inequality

$$
\|K(r)[\Psi]\|_{\mathcal{H}}^{2}=\|[\mathcal{U}(r) \Psi]\|_{\mathcal{H}}^{2}=\langle\mathcal{U}(r) \Psi, R \mathcal{U}(r) \Psi\rangle_{\mathcal{H}^{\prime}} \leq\|\mathcal{U}(r) \Psi\|_{\mathcal{H}^{\prime}}\|R \mathcal{U}(r) \Psi\|_{\mathcal{H}^{\prime}} \leq\|\Psi\|_{\mathcal{H}^{\prime}}^{2}
$$

Thus, for any $n$

$$
\|K(s)[\Psi]\|_{\mathcal{H}} \leq\left.\|[\Psi]\|\right|_{\mathcal{H}} ^{1-2^{-n}}\|\Psi\|_{\mathcal{H}^{\prime}}^{2-n}
$$

and taking the limit $n \rightarrow \infty$

$$
\frac{\|K(s)[\Psi]\|_{\mathcal{H}}}{\|[\Psi]\|_{\mathcal{H}}} \leq 1
$$

Taking the supremum over $[\Psi] \neq 0$ shows that $K(s)$ is bounded in norm by one, i.e. $s \rightarrow K(s)$ is a contraction semigroup on $\mathcal{H}$. Note that there are two Hilbert space norms involved in (2.38) so that unitarity of $\mathcal{U}(r)$ alone would not suffice to establish (2.39).

Next, by assumption $\mathcal{U}(s)$ is reflection continuous whence for $\Psi \in \mathcal{H}$

$$
\begin{aligned}
\left\|\left[K(s)-1_{\mathcal{H}}\right][\Psi]\right\|^{2} & =\left\langle\Psi,\left[\mathcal{U}(2 s)-2 \mathcal{U}(s)+1_{\mathcal{H}^{\prime}}\right] R \Psi\right\rangle_{\mathcal{H}^{\prime}} \\
& \leq\left|\left\langle\Psi,\left[\mathcal{U}(2 s)-1_{\mathcal{H}^{\prime}}\right] R \Psi\right\rangle_{\mathcal{H}^{\prime}}\right|+2\left|\left\langle\Psi,\left[\mathcal{U}(s)-1_{\mathcal{H}^{\prime}}\right] R \Psi\right\rangle_{\mathcal{H}^{\prime}}\right|
\end{aligned}
$$

tends to zero as $s \rightarrow 0$. Hence the contraction semi-group is continuous and therefore has a selfadjoint generator $H$ by the Hille-Yosida theorem [72].

Finally, we define $\Omega:=[1]$ where $1 \in \mathcal{H}^{\prime}$ is the constant function equal to unity which we may think of as $W[F]_{F=0}$. Then evidently

$$
\left.K(s) \Omega=[\mathcal{U}(s) W[F]]_{F=0}\right]=\left[W\left[T_{s} F\right]_{F=0}\right]=[1]=\Omega
$$


so that $\Omega$ is a vacuum for $H$.

For the sake of completeness, we show that the vacuum is unique if if $\mu$ is time-clustering, i.e.

$$
\lim _{s \rightarrow \infty} \mu\left(W[F]^{*} W\left[T_{s} F^{\prime}\right]\right)=\mu\left(W[F]^{*}\right) \mu\left(W\left[F^{\prime}\right]\right)=\langle W[F], 1\rangle_{\mathcal{H}^{\prime}}\left\langle 1, W\left[F^{\prime}\right]\right\rangle_{\mathcal{H}^{\prime}}
$$

Using that the span of the $W[F]$ is dense, property (2.42) says that, as $s \rightarrow \infty$, the operator $\mathcal{U}(s)$ becomes the projection operator $P=1\langle 1, .\rangle_{\mathcal{H}^{\prime}}$ on $\mathcal{H}^{\prime}$ in the weak operator topology. Now suppose that $K(s) \Omega=\Omega$. We find $\Omega^{\prime} \in \mathcal{H}^{\prime}$ such that $\left[\Omega^{\prime}\right]=\Omega$. It follows

$$
\left[K(s)-1_{\mathcal{H}}\right] \Omega=\left[\left[\mathcal{U}(s)-1_{\mathcal{H}^{\prime}}\right] \Omega^{\prime}\right]=0
$$

i.e. $\left[\mathcal{U}(s)-1_{\mathcal{H}^{\prime}}\right] \Omega^{\prime} \in \mathcal{N}$ for any $s$. Thus, if $\mu$ clusters, then by taking the limit in (2.43) we find that $\left\langle 1, \Omega^{\prime}\right\rangle 1-\Omega^{\prime} \in \mathcal{N}$, i.e. $\Omega:=\left[\Omega^{\prime}\right] \propto[1]$ and the vacuum is unique (up to a phase when normalised).

Remark:

The Osterwalder-Schrader reconstruction of the data $(\mathcal{H}, \Omega, H)$ just relies on the time reflection and translation invariance, time continuity and time reflection positivity. Neither is anything being said about the existence of a "mass gap" nor does OS reconstruction rely on it, not even when restricting to QFT in Minkowski space. For QFT in Minkowski space, by a mass gap is meant a strictly positive infimum in the support, without zero, of the Källen-Lehmann spectral measure for the 2-point function and as such is a very "Minkowskian" concept. Such a mass gap is important in order to develop Haag-Ruelle scattering theory (rigorous collision theory) and in particular for constructive Yang-Mills theory it would be an indirect proof of confinement. In our general theory setting, which in principle encompasses quantum gravity in suitable gauges, it is not even expected that the 2-point function is related in such a simple way to a suitable notion of mass since the quantum gravity vacuum would presumably describe a spacetime with a degenerate geometry expectation value. Hence, such and related questions can meaningfully be asked only when adding further structural elements (e.g. correlation functions in states describing a semiclassical geometry).

\subsection{OS data reconstruction and OS measure construction are inverses}

We wish to demonstrate that OS construction (i.e. computing the automatically reflection positive Wiener measure from OS data) and OS reconstruction (i.e. computing OS data from a reflection positive measure) are inverse processes. Recall from our preliminary remarks that is implicit in our

assumptions that, if necessary, we have recast our formulation into a form such that when starting with the OS data, the sharp time zero Weyl elements $w[f]$ are well defined and the $w[f] \Omega$ lie dense in the canonical Hilbert space $\mathcal{H}$. Likewise, starting with the OS measure, if the sharp time $W\left[\delta_{0} f\right]$ are not well defined as $\mu$ measurable and integrable functions and/or the $\left[W\left[\delta_{0} f\right]\right]$ do not lie dense in the OS Hilbert space, we make use of our assumption spelled out in the preliminaries that the OS Hilbert space $\mathcal{H}$ admits an Abelian $C^{*}$-subalgebra $\mathfrak{B}$ of $\mathcal{B}(\mathcal{H})$ such that $\mathfrak{B} \Omega$ is dense. We then reformulate the OS measure $\mu$ in terms of the Weyl elements $w[f]$ generating $\mathfrak{B}$. Then the corresponding functions of the history field $\Phi$ given by $W\left[\delta_{s} f\right]_{\Phi}=w[f]_{\phi \rightarrow \Phi(s)}$ exist as $\mu$-integrable functions and allow to construct the generating functional of Schwinger functions. It is also being understood that we have reformulated $\mu$ in this way, if necessary.

\subsubsection{Reproduction of OS data}

We first start with OS data $(\mathcal{H}, \Omega, H)$ and construct the Wiener measure $\mu$ out of them according to section 2.2. We ask, whether the reconstruction algorithm of section 2.3 recovers these data.

Concerning the null space, using the same notation as in (2.23), (2.24), recall

$$
\|[\Psi]\|_{V / \mathcal{N}}^{2}=\left\|\sum_{I} z_{I} e^{-\beta_{1}^{I} H} w\left[f_{1}^{I}\right] e^{-\left(\beta_{2}^{I}-\beta_{1}^{I}\right) H} . . e^{-\left(\beta_{N^{I}}^{I}-\beta_{N^{I}-1}^{I}\right) H} w\left[f_{N^{I}}^{I}\right] \Omega\right\|_{\mathcal{H}}^{2}
$$


Consider the vector in $\mathcal{H}$

$$
\psi:=\sum_{I} z_{I} e^{-\beta_{1}^{I} H} w\left[f_{1}^{I}\right] e^{-\left(\beta_{2}^{I}-\beta_{1}^{I}\right) H} . . e^{-\left(\beta_{N^{I}}^{I}-\beta_{N^{I}-1}^{I}\right) H} w\left[f_{N^{I}}^{I}\right] \Omega
$$

that appears in (2.44). Since by assumption 1. above the finite linear span of the $w[f] \Omega$ lies dense in $\mathcal{H}$, for any $\epsilon>0$ we find $\psi^{\epsilon}=\sum_{J} c_{J} w\left[g^{J}\right] \Omega$ such that $\left\|\psi-\psi^{\epsilon}\right\|_{\mathcal{H}}<\epsilon$. Consider the corresponding $\Psi^{\epsilon}=\sum_{J} c_{J} W\left[G^{J}\right], G^{J}=\delta_{0} g^{J}$. Then by the same calculation as in (2.24)

$$
\left\|[\Psi]-\left[\Psi^{\epsilon}\right]\right\|_{\overline{V / \mathcal{N}}}^{2}=\left\|\psi-\psi^{\epsilon}\right\|_{\mathcal{H}}^{2}<\epsilon^{2}
$$

Since the scalar product on $\mathcal{H}$ is non-degenerate, this demonstrates that the equivalence class of $[\Psi]$ can be labelled by representatives which lie in the closure of the span of the $W[F]$ with $F=\delta_{0} f$. This suggests to define the densely defined embedding

$$
E: \mathcal{H} \rightarrow V, \psi=\sum_{I} z_{I} w\left[f^{I}\right] \Omega \mapsto \Psi=\sum_{I} z_{I} W\left[\delta_{0} f^{I}\right]
$$

It follows that $E(\Omega)=1$ is the constant function equal to unity and the scalar products are isometric $\|[E(\psi)]\|_{\overline{V / \mathcal{N}}}=\|\psi\|_{\mathcal{H}}$, i.e. $[E(\psi)]$ can be identified with $\psi$. This shows that the equivalence classes of vectors with respect to the reflection positive inner product are precisely labelled by vectors in $\mathcal{H}$ and $E$ defines a section in the Hilbert bundle $\pi: V \rightarrow \mathcal{H}$ with projection $\pi()=.[$.$] , that is,$ $\pi \circ E=\operatorname{id}_{\mathcal{H}}$ and $V, \mathcal{H}$ are respectively total space and base of the bundle. We conclude $\overline{V / \mathcal{N}} \equiv \mathcal{H}$.

It follows then from (2.44) and (2.45) that for $F=\left(\delta_{\beta_{k}} f_{k}\right)_{k=1}^{N}, 0<\beta_{1}<. .<\beta_{N}$

$$
[W[F]] \equiv e^{-\beta_{1} H} w\left[f_{1}\right] e^{-\left(\beta_{2}-\beta_{1}\right) H} w\left[f_{2}\right] . . e^{-\left(\beta_{N}-\beta_{N-1}\right) H} w\left[f_{N}\right] \Omega
$$

We compute the contraction semi-group for $\psi=\sum_{I} z_{I} w\left[f^{I}\right] \Omega$ from (2.48),

$$
K(s) \psi \equiv K(s)[E(\psi)]=[\mathcal{U}(s) E(\psi)]=\left[\sum_{I} z_{I} W\left[\delta_{s} f^{I}\right]\right] \equiv \sum_{I} z_{I} e^{-s H} w\left[f^{I}\right] \Omega=e^{-s H} \psi
$$

whence $K(s)=e^{-s H}$ indeed.

In conclusion, we have shown that the Wiener measure constructed from the data $(\mathcal{H}, \Omega, H)$ has precisely those data as OS data.

\subsubsection{Reproduction of the OS measure}

Conversely, suppose that we are given an OS measure $\mu$. Does the Wiener measure $\mu^{\prime}$ constructed from its OS data $\left(\mathcal{H}=\overline{V / \mathcal{N}}, \Omega=[1], H=-[d / d s]_{s=0} K(s)\right)$ coincide with the measure $\mu$ that we started from? To define $\mu^{\prime}$ we must actually first define the Weyl operators $w[f]$ on the Hilbert space $\mathcal{H}$. According to introduction to this subsection, it is meaningful to define them densely $\left(G=\left(\delta_{\beta_{k}} g_{k}\right)_{k=1}^{N}, 0<\beta_{1}, . ., \beta_{N}\right.$ is of discrete and positive time support) by

$$
w[f][W[G]]:=\left[W\left[F_{f}^{0}\right] W[G]\right], \quad F_{f}^{0}:=\delta_{0} f
$$

In general, we set $F_{f}^{s}:=\delta_{s} f$. Notice the identities $T_{r} F_{f}^{s}=F_{f}^{s+r}, \theta F_{f}^{s}=F_{f}^{-s}$. Recall also $K(s)[\Psi]=[\mathcal{U}(s) \Psi]$. Definition (2.50) is independent of the representative because suppose that $[\Psi]=0$ with $\Psi$ in the finite linear span of the $W[F]$ with $F$ of positive time support then

$$
\begin{aligned}
\|w[f][\Psi]\|_{\mathcal{H}}^{2} & =\left\langle W\left[F_{f}^{0}\right] \Psi, R \cdot\left(W\left[F_{f}^{0}\right] \Psi\right)\right\rangle_{\mathcal{H}^{\prime}}=\left\langle W\left[F_{f}^{0}\right] \Psi, W\left[F_{f}^{0}\right](R \cdot \Psi)\right\rangle_{\mathcal{H}^{\prime}} \\
& =\left\langle w[f]^{*} w[f][\Psi],[\Psi]\right\rangle_{\mathcal{H}} \leq\left\|w[f]^{*} w[f][\Psi]\right\|_{\mathcal{H}}\|[\Psi]\|_{\mathcal{H}}=0
\end{aligned}
$$

by the Cauchy-Schwarz inequality. We used that $\theta \cdot \delta_{0}=\delta_{0}$. Indeed this was very crucial because if representing $w[f]$ in terms of some $W[F]$ had also involved strictly positive time support in $F$ then 
we could not have made this conclusion because $R W[F]=W[\theta F] R$ and $W[\theta F]^{*}$ does not even preserve $V$.

We compute for $F$ of discrete time support at $\beta_{1}<. .<\beta_{N}$ and with $F_{k}^{s}:=F_{f_{k}}^{s}$

$$
\begin{aligned}
& \mu^{\prime}(W[F])=\left\langle\Omega, w\left[f_{N}\right] K\left(\beta_{N}-\beta_{N-1}\right) . . K\left(\beta_{2}-\beta_{1}\right) w\left[f_{1}\right] \Omega\right\rangle_{\mathcal{H}} \\
& =\left\langle\Omega, w\left[f_{N}\right] K\left(\beta_{N}-\beta_{N-1}\right) . . K\left(\beta_{2}-\beta_{1}\right)\left[W\left[F_{1}^{0}\right] \cdot 1\right]\right\rangle_{\mathcal{H}} \\
& =\left\langle\Omega, w\left[f_{N}\right] K\left(\beta_{N}-\beta_{N-1}\right) . . K\left(\beta_{3}-\beta_{2}\right) w\left[f_{2}\right]\left[\mathcal{U}\left(\beta_{2}-\beta_{1}\right) W\left[F_{1}^{0}\right] \cdot 1\right]\right\rangle_{\mathcal{H}} \\
& =\left\langle\Omega, w\left[f_{N}\right] K\left(\beta_{N}-\beta_{N-1}\right) . . K\left(\beta_{3}-\beta_{2}\right) w\left[f_{2}\right]\left[W\left[F_{1}^{\beta_{2}-\beta_{1}}\right]\right]\right\rangle_{\mathcal{H}} \\
& =\left\langle\Omega, w\left[f_{N}\right] K\left(\beta_{N}-\beta_{N-1}\right) . . K\left(\beta_{3}-\beta_{2}\right)\left[W\left[F_{2}^{0}\right] W\left[F_{1}^{\beta_{2}-\beta_{1}}\right]\right]\right\rangle_{\mathcal{H}} \\
& =\left\langle\Omega, w\left[f_{N}\right] K\left(\beta_{N}-\beta_{N-1}\right) . . K\left(\beta_{4}-\beta_{3}\right) w\left[f_{3}\right]\left[W\left[F_{2}^{\beta_{3}-\beta_{2}}\right] W\left[F_{1}^{\beta_{3}-\beta_{1}}\right]\right]\right\rangle_{\mathcal{H}} \\
& =\left\langle[1],\left[W\left[F_{N}^{\beta_{N}-\beta_{N}}\right] \ldots W\left[F_{1}^{\beta_{N}-\beta_{1}}\right]\right]\right\rangle_{\mathcal{H}} \\
& =\left\langle 1, R W\left[F_{N}^{\beta_{N}-\beta_{N}}\right] \ldots W\left[F_{1}^{\beta_{N}-\beta_{1}}\right]\right\rangle_{\mathcal{H}^{\prime}} \\
& =\left\langle 1, W\left[F_{N}^{\beta_{N}-\beta_{N}}\right] \ldots W\left[F_{1}^{\beta_{1}-\beta_{N}}\right]\right\rangle_{\mathcal{H}^{\prime}} \\
& =\left\langle 1, \mathcal{U}\left(-\beta_{N}\right) W\left[F_{N}^{\beta_{N}}\right] \ldots W\left[F_{1}^{\beta_{1}}\right]\right\rangle_{\mathcal{H}^{\prime}} \\
& =\left\langle\mathcal{U}\left(\beta_{N}\right) \cdot 1, W\left[F_{N}^{\beta_{N}}\right] \ldots W\left[F_{1}^{\beta_{1}}\right]\right\rangle_{\mathcal{H}^{\prime}} \\
& =\langle 1, W[F]\rangle_{\mathcal{H}^{\prime}}=\mu(W[F])
\end{aligned}
$$

thus indeed the Wiener measure coincides with $\mu$.

\section{Brief review of path integral renormalisation}

In constructive QFT one works in the Euclidian framework and seeks to construct a measure $\mu$ on the history space of fields $\Phi \in \Gamma$ satisfying the Osterwalder-Schrader axioms. The construction uses both an infrared (IR) cut-off $R$ and an ultraviolet (UV) cut-off $\epsilon$. In principle, one can distinguish between spatial cut-offs $R, \epsilon$ and temporal cut-offs $T, \delta$ although one often identifies them $\epsilon=\delta$ and $T=R$. We will keep the distinction, because in the Hamiltonian framework, time is always a continuous parameter and therefore is treated differently. Making the distinction between spatial and temporal will enable us to simplify the match between the path integral and Hamiltonian renormalisation later on.

In order to understand the role of $T, \delta, R, \epsilon$ and how they find their way into the Euclidian formulation, we start from the observation, that the measure $\mu$ is completely specified by its generating functional $\mu(W[F])$ where $W[F, \Phi]$ involves the smearing field $F$ of the history quantum field $\Phi$. The IR cut-offs are introduced simply by restricting the support of $F$ to $[0, T] \times \sigma_{R}$ where $\sigma_{R}$ is a compact submanifold of $\sigma$, for instance $[0, R]^{D}$ if $\sigma=\mathbb{R}^{D}$. Thus, such smearing functions probe $\Phi$ only on a compact submanifold of $M=\mathbb{R} \times \sigma$. The UV cut-offs are introduced by restricting to smearing functions that in addition probe $\Phi$ only with finite temporal and spatial resolution $\delta$ and $\epsilon$ respectively. For instance, such a function could be constant on each cell of a lattice in $[-T, T] \times \sigma_{R}$ with cell size of the order of $\delta \epsilon^{D}$ and thus is completely specified by its value on the lattice vertices. In what follows, we will trade the numbers $\delta, \epsilon$ for the numbers of $N, M$ which indicate the number of vertices of the lattice in the temporal and each spatial direction respectively. This means $\delta=\delta_{T, N}=\frac{T}{N}, \epsilon=\epsilon_{R, M}=\frac{R}{M}$ if we consider a regular hypercubic lattice in $\mathbb{R}^{D+1}$. In general, the relation among the $T, N, \delta$ and the $R, M, \epsilon$ maybe more involved.

To have something concrete in mind, for a scalar field on $\mathbb{R}^{D+1}$ such a compactly supported 
function of the indicated resolution could be given by

$$
\begin{aligned}
F_{T, N, R, M}(t, x) & =\sum_{n \in \mathbb{Z}_{N}, m \in \mathbb{Z}_{M}^{D}} \hat{F}_{T, N, R, M}(n, m) \chi_{n \delta_{T, N}, m \epsilon_{R, M}}(t, x) \\
\chi_{n \delta_{T, N}, m \epsilon_{R, M}}(t, x) & =\chi_{\left[n \delta_{T, N},(n+1) \delta_{T, N}\right)}(t) \prod_{a=1}^{D} \chi_{\left[m^{a} \epsilon_{R, M},\left(m^{a}+1\right) \epsilon_{R, M}\right)}\left(x^{a}\right)
\end{aligned}
$$

where $\mathbb{Z}_{N}:=\{0,1, . ., N-1\}, \mathbb{Z}_{M}:=\{0,1, . ., M-1\}$. Note that indeed the coefficient $\hat{F}_{T, N, R, M}(n, m)$ in (3.1) is the value of $F_{T, N, R, M}(t, x)$ at $(t, x)=\left(n \delta_{T, N}, m \epsilon_{R, M}\right)$.

We want to distinguish between the continuum function $F_{T, N, R, M}$ and its restriction to the lattice parametrised by $T, N, R, M$. Let $L_{T, R}$ be the space of compactly supported continuum smearing functions and $L_{T, N, R, M}$ the space of lattice smearing functions. In the example above, $L_{T, N, R, M}$ is simply the set of finite sequences $\hat{F}_{T, N, R, M}(n, m), n \in \mathbb{Z}_{N}, m \in \mathbb{Z}_{M}^{D}$. Then we obtain maps

$$
\begin{aligned}
E_{T, N, R, M}: & L_{T, R} \rightarrow L_{T, N, R, M} \\
& \left(E_{T, N, R, M} \cdot F_{T, R}\right)(n, m):=F_{T, R}\left(n \delta_{T, N}, m \epsilon_{R, M}\right) \\
I_{T, N, R, M}: & L_{T, N, R, M} \rightarrow L_{T, R} \\
& \left(I_{T, N, R, M} \hat{F}_{T, N, R, M}\right)(t, x):=\sum_{n \in \mathbb{Z}_{N}, m \in \mathbb{Z}_{M}^{D}} \hat{F}_{T, N, R, M}(n, m) \chi_{n \delta_{T, N}, m \epsilon_{R, M}}(t, x)
\end{aligned}
$$

In fact, in this concrete situation the map $E_{T, N, R, M}$ is redundant because the spaces $L_{T, N, R, M}, L_{T, R}$ here carry the additional structure of an inner product so that instead of $E_{T, N, R, M}$ we can consider $I_{T, N, R, M}^{\dagger}$. This has the advantage of introducing less structure at the price of keeping the inner products. We keep $E_{T, N, R, M}$ as a separate entity for more general situations in which natural inner products are not available.

Note that while the evaluation map $E_{T, N, R, M}$ is canonical and theory independent (it just requires the knowledge of the relation between the integers $(n, m)$ and the points of the lattice), the injection map $I_{T, N, R, M}$ is not canonical and does depend on the type of the theory under consideration and is of the above form only for a scalar field. For other types of fields it will assume a different form, but still such a map can always be invented. We will restrict the choice of $I_{T, N, R, M}$ by insisting that

$$
E_{T, N, R, M} \circ I_{T, N, R, M}=\operatorname{id}_{L_{T, N, R, M}}
$$

which is satisfied for the choice (3.2). It means that the discrete information contained in a lattice function can be fully recovered by evaluation after injection. It will be the choice of the injection map that will determine the coarse graining map and therefore the whole renormalisation procedure. As it happens, for the concrete choice (3.2) condition (3.3) is also satisfied if we replace $I_{T, N, R, M}^{\dagger}$ for $E_{T, N, R, M}$. This means that $I_{T, N, R, M}$ is there an isometric embedding.

The formula that defines $W\left[F_{T, R}, \Phi_{T, R}\right]$, that is, the pairing between $F_{T, R} \in L_{T, R}$ and $\Phi_{T, R} \in$ $\Gamma_{T, R}$ in the continuum induces a discretised lattice field $\hat{\Phi}_{T, N, R, M}$ and a corresponding pairing via

$$
W_{T, R}\left[I_{T, N, R, M} \hat{F}_{T, N, R, M}, \Phi_{T, R}\right]=: W_{T, N, R, M}\left[\hat{F}_{T, N, R, M}, \hat{\Phi}_{T, N, R, M}\right]
$$

For instance, for a scalar field we have

$$
\begin{aligned}
W_{T, R}\left[F_{T, R}, \Phi_{T, R}\right] & =\exp \left(i\left\langle F_{T, R}, \Phi_{T, R}\right\rangle\right) \\
\left\langle F_{T, R}, \Phi_{T, R}\right\rangle & :=\int_{\mathbb{R}} d t \int_{\sigma} d^{D} x F_{T, R}(t, x) \Phi(t, x) \\
& \Rightarrow \exp \left(i\left\langle\hat{F}_{T, N, R, M}, \hat{\Phi}_{T, N, R, M}\right\rangle\right), \\
W_{T, N, R, M}\left[\hat{F}_{T, N, R, M}, \hat{\Phi}_{T, N, R, M}\right] & =\hat{\Phi}_{T, N, R, M}(n, m), \\
\left\langle\hat{F}_{T, N, R, M}, \hat{\Phi}_{T, N, R, M}\right\rangle & :=\epsilon_{R, M}^{D} \delta_{T, N} \sum_{n, m} \hat{F}_{T, N, R, M}(n, m) \hat{\Phi}_{T, N, M}(n, m) \\
\hat{\Phi}_{T, N, R,} & :\left\langle\chi_{n \delta_{T, N}, m \epsilon_{R, M}}, \Phi\right\rangle
\end{aligned}
$$


The discretised history field can now be used to define a probability measure $\mu_{T, N, R, M}$ on the finite dimensional space $\Gamma_{T, N, R, M}$. In case of a scalar field we have obviously $\Gamma_{T, N, R, M}=\mathbb{R}^{N M^{D}}$ and one will pick a measure on $\Gamma_{T, N, R, M}$ which is of the form:

(Lebesgue measure) times (damping factor) times (normalisation constant). The damping factor is usually motivated from the classical theory and is often the exponential of the negative of the discretised Euclidian version of the action. Thus, to have something concrete in mind for a scalar field we consider a measure of the form

$$
\begin{aligned}
d \mu_{T, N, R, M}\left(\hat{\Phi}_{T, N, R, M}\right) & =\frac{\rho_{T, N, R, M}\left(\hat{\Phi}_{T, N, R, M}\right)}{Z_{T, N, R, M}} d^{N M^{D}} \hat{\Phi}_{T, N, R, M}, \\
d^{N M^{D}} \hat{\Phi}_{T, N, R, M} & :=\prod_{n \in \mathbb{Z}_{N}, m \in \mathbb{Z}_{M}^{D}} d \hat{\Phi}(n, m)
\end{aligned}
$$

At this point we will not be specific about the choice of these three ingredients, but point out, that the discretisation of the Euclidian action, and thus the choice of $\mu_{T, N, R, M}$ is of course far from unique, it is actually ambiguous. This is the case even if we impose that the formal continuum limit of $\rho_{T, N, R, M}$ as $N, M \rightarrow \infty$ should be $\exp \left(-S_{T, R}[\Phi]\right)$ where $S_{T, R}[\Phi]$ is the continuum Euclidian action restricted to $[0, T] \times \sigma_{R}$. In our concrete situation, such a discretisation could be chosen for instance as

$$
S_{T, N, R, M}\left[\hat{\Phi}_{T, N, R, M}\right]:=S\left[I_{T, N, R, M} \circ \frac{\hat{\Phi}_{T, N, R, M}}{\delta_{T, N} \epsilon_{R, M}^{D}}\right]
$$

where the division by the cell volume reproduced the value $E_{T, N, R, M} \cdot \Phi$ in the continuum limit. However, whatever ambiguous choice of discretisation one has made, we insist that the same discretisation procedure has been applied for all values of $T, N, R, M$. For instance, if one has chosen to approximate a Laplacian by using only next neighbour field differences for one quadruple $\left(T_{0}, N_{0}, M_{0}, R_{0}\right)$ then one does it for all $(T, N, R, M)$ rather than arbitrarily taking also next to next neighbour differences into account for other values of $(T, N, R, M)$.

In this way we have produced a family of measures $\mu_{T, N, R, M}$ parametrised by $T, R, N, M$ where these numbers can all be arbitrarily large as long as they are finite. Each member of the family is specified by its corresponding generating functional

$$
\mu_{T, N, R, M}\left(W_{T, N, R, M}\left[\hat{F}_{T, N, R, M}\right]\right)
$$

Now suppose that a continuum measure $\mu_{T, R}$ would exist whose generating functional is $\mu_{T, R}\left(W_{T, R}\left[F_{T, R}\right]\right)$ with $F_{T, R} \in L_{T, R}$. In view of (3.4) the injection map defines its cylindrical projections

$$
\mu_{T, R}^{N, M}\left(W_{T, N, R, M}\left[\hat{F}_{T, N, R, M}\right]\right):=\mu_{T, R}\left(W_{T, R}\left[I_{T, N, R, M} \cdot \hat{F}_{T, N, R, M}\right]\right)
$$

i.e. by restricting the smearing functions to be those of finite resolution.

This is the point where the coarse graining map comes into play. Namely we can combine injection and evaluation maps to produce the maps

$$
I_{T, N \rightarrow N^{\prime}, R, M \rightarrow M^{\prime}}:=E_{T, N^{\prime}, R, M^{\prime}} \circ I_{T, N, R, M}: L_{T, N, R, M} \rightarrow L_{T, N^{\prime}, R, M^{\prime}}
$$

where $N, N^{\prime}, M, M^{\prime}$ are arbitrary. Useful combinations of these numbers are $N^{\prime}=2^{n} N, M^{\prime}=$ $2^{m} M, n, m \in \mathbb{N}_{0}$ because they correspond to viewing a function defined on the coarse lattice $(T, N, R, M)$ as a function on the finer lattice $\left(T, 2^{n} N, R, 2^{m} M\right)$ of which the former is a sublattice. We will call them coarse graining maps in this case. We will further impose the following restriction on the choice of the injection maps

$$
I_{T, 2^{n} N, R, 2^{m} M} \circ I_{T, N \rightarrow 2^{n} N, R, M \rightarrow 2^{m} M}=I_{T, N, R, M}
$$

which is satisfied for the choice (3.2). It means that considering a lattice function as a special continuum function is independent on what sublattice it is actually defined. To see this, we compute 
for $k \in \mathbb{Z}_{2^{n} N}, l \in \mathbb{Z}_{2^{m} M}^{D}$

$$
\begin{aligned}
{\left[I_{T, N \rightarrow 2^{n} N, R, M \rightarrow 2^{m} M} \cdot \hat{F}_{T, N, R, M}\right](k, l) } & =\sum_{k^{\prime} \in \mathbb{Z}_{N}, l^{\prime} \in \mathbb{Z}_{M}^{D}} \chi_{k^{\prime} \delta_{T, N}, l^{\prime} \epsilon_{R, M}}\left(k \delta_{T, 2^{n} N}, l \epsilon_{R, 2^{m}}\right) \hat{F}_{T, N, R, M}\left(k^{\prime}, l^{\prime}\right) \\
& =\hat{F}_{T, N, R, M}\left(\left\lfloor 2^{-n} k\right\rfloor,\left\lfloor 2^{-m} l\right\rfloor\right)
\end{aligned}
$$

where the bracket is the Gauss bracket (separately for each argument), i.e. $\left\lfloor 2^{-m} l\right\rfloor^{a}:=\left\lfloor 2^{-m} l^{a}\right\rfloor$. Accordingly

$$
\begin{aligned}
& \left(I_{T, 2^{n} N, R, 2^{m} M} \circ I_{T, N \rightarrow 2^{n} N, R, M \rightarrow 2^{m} M} \cdot \hat{F}_{T, N, R, M}\right)(t, x) \\
& =\sum_{k \in \mathbb{Z}_{2^{n} N}, l \in \mathbb{Z}_{2^{m}}^{D} M} \chi_{k \delta_{T, 2^{n} N}, l \epsilon_{R, 2} m_{M}}(t, x) \hat{F}_{T, N, R, M}\left(\left\lfloor 2^{-n} k\right\rfloor,\left\lfloor 2^{-m} l\right\rfloor\right) \\
& =\sum_{k^{\prime} \in \mathbb{Z}_{N}, l^{\prime} \in \mathbb{Z}_{M}^{D}} \hat{F}_{T, N, R, M}\left(k^{\prime}, l^{\prime}\right) \sum_{k \in \mathbb{Z}_{2^{n} N}, l \in \mathbb{Z}_{2^{m}}^{D}{ }_{M} ;\left\lfloor k 2^{-n}\right\rfloor=k^{\prime} ;\left\lfloor 2^{-m} l\right\rfloor=l^{\prime}} \chi_{k \delta_{T, 2^{n} N}, l \epsilon_{R, 2^{m}} M}(t, x) \\
& =\sum_{k^{\prime} \in \mathbb{Z}_{N}, l^{\prime} \in \mathbb{Z}_{M}^{D}} \hat{F}_{T, N, R, M}\left(k^{\prime}, l^{\prime}\right) \sum_{k=k^{\prime}+r, l=l^{\prime}+s ; r \in\left\{0, . ., 2^{n}-1\right\} ; s \in\left\{0, . ., 2^{m}-1\right\}^{D}} \chi_{k \delta_{T, 2^{n} N}, l \epsilon_{R, 2^{m}} M}(t, x) \\
& =\sum_{k^{\prime} \in \mathbb{Z}_{N}, l^{\prime} \in \mathbb{Z}_{M}^{D}} \hat{F}_{T, N, R, M}\left(k^{\prime}, l^{\prime}\right) \chi_{k^{\prime} \delta_{T, N}, l^{\prime} \epsilon_{R, M}}(t, x) \\
& =\left(I_{T, N, R, M} \cdot \hat{F}_{T, N, R, M}\right)(t, x)
\end{aligned}
$$

as claimed.

As a consequence, we have the following consistency condition among the coarse graining maps

$$
\begin{aligned}
& I_{T, 2^{n} N \rightarrow 2^{n+n^{\prime}} N, R, 2^{m} M \rightarrow 2^{m+m^{\prime}} M} \circ I_{T, N \rightarrow 2^{n} N, R, M \rightarrow 2^{m} M} \\
= & E_{T, 2^{n+n^{\prime}} N, R, 2^{m+m^{\prime}} M} \circ\left[I_{T, 2^{n} N, R, 2^{m} M} \circ I_{T, N \rightarrow 2^{n} N, R, M \rightarrow 2^{m} M}\right]=E_{T, 2^{n+n^{\prime}} N, R, 2^{m+m^{\prime}} M} \circ I_{T, N, R, M} \\
= & I_{T, N \rightarrow 2^{n+n^{\prime}} N, R, M \rightarrow 2^{m+m^{\prime}} M}
\end{aligned}
$$

It means that coarse graining can be done in steps over arbitrary intermediate sublattices.

For the cylindrical projections of $\mu_{T, R}$ this has the following consequence called cylindrically consistency

$$
\begin{aligned}
& \mu_{T, R}^{N, M}\left(W_{T, N, R, M}\left[F_{T, N, R, M}\right]\right)=\mu_{T, R}\left(W_{T, R}\left[I_{T, N, R, M} \cdot \hat{F}_{T, N, R, M}\right]\right) \\
= & \mu_{T, R}\left(W_{T, R}\left[I_{T, 2^{n} N, R, 2^{m} M} \cdot I_{T, N \rightarrow 2^{n} N, R, M \rightarrow 2^{m} M} \circ \hat{F}_{T, N, R, M}\right]\right) \\
= & \mu_{T, R}^{2^{n} N, 2^{m} M}\left(W_{T, 2^{n} N, R, 2^{m} M}\left[I_{T, N \rightarrow 2^{n} N, R, M \rightarrow 2^{m} M} \cdot \hat{F}_{T, N, R, M}\right]\right)
\end{aligned}
$$

This means that a necessary condition for a projective family $(M, N) \mapsto\left(\mu_{T, R}^{M, N}\right)$ to qualify as the cylindrical projections of a continuum measure, is that the family is cylindrically consistent. In suitable mathematical contexts, depending on the details of the theory under consideration, the condition is also sufficient [9]. Therefore, cylindrical consistency is a very useful criterion in order to detect the continuum limit of a projective family.

Renormalisation is now a construction principle to actually derive a cylindrically consistent family from a given family $\mu_{T, N, R, M}^{(0)}$, that was merely defined as above, by employing possibly natural but still ad hoc discretisation prescriptions. We construct the sequence $\left(\mu_{T, N, R, M}^{(n)}\right)_{n \in \mathbb{N}_{0}}$ inductively from $\mu_{T, N, R, M}^{(0)}$ by a block spin transformation

$$
\mu_{T, N, R, M}^{(n+1)}\left(W_{T, N, R, M}\left[\hat{F}_{T, N, R, M}\right]\right):=\mu_{T, 2 N, R, 2 M}^{(n)}\left(W_{T, 2 N, R, 2 M}\left[I_{T, N \rightarrow 2 N, R, M \rightarrow 2 M} \cdot \hat{F}_{T, N, R, M}\right]\right)
$$

Note that (3.16) indeed defines an entire new family of measures from the old one, because 1 . each measure is completely defined in terms of its generating functional and 2. one performs (3.16) 
coherently for all $M, N$. The sequence of measures thus defined may or may not converge (here we use the notion of pointwise convergence on the $W_{T, N, R, M}\left[F_{T, N, R, M}\right]$ ). If it or at least a subsequence converges, we call the limit a fix point family $\mu_{T, N, R, M}^{*}$. The fix point family is cylindrically consistent in the sense

$$
\begin{aligned}
& \mu_{T, 2 N, R, 2 M}^{*}\left(W_{T, 2 N, R, 2 M}\left[I_{T, N \rightarrow 2 N, R, M \rightarrow 2 M} \cdot \hat{F}_{T, N, R, M}\right]\right) \\
= & \lim _{n \rightarrow \infty} \mu_{T, 2 N, R, 2 M}^{(n)}\left(W_{T, 2 N, R, 2 M}\left[I_{T, N \rightarrow 2 N, R, M \rightarrow 2 M} \cdot \hat{F}_{T, N, R, M}\right]\right) \\
= & \lim _{n \rightarrow \infty} \mu_{T, N, R, M}^{(n+1)}\left(W_{T, N, R, M}\left[\hat{F}_{T, N, R, M}\right]\right)=\mu_{T, N, R, M}^{*}\left(W_{T, N, R, M}\left[\hat{F}_{T, N, R, M}\right]\right)
\end{aligned}
$$

and thus defines a continuum measure $\mu_{T, R}^{*}$ under the afore mentioned conditions.

Note that the fix point family of course depends on the parameters $N, M$. The measure $\mu_{T, N, R, M}^{*}$ at given $N, M$ is the continuum measure, but restricted to observables, that is, field probes $F_{T, N, R, M}=I_{T, N, R, M} \cdot \hat{F}_{T, N, R, M}$, of finite resolution. In the physics jargon customary in renormalisation, these are the so-called perfect measures (or perfect actions if one prefers to think in terms of actions rather than measures). The terminology intends to express the fact that although one measures at finite resolution, the measurements are still described by the continuum theory [75-77] .

Finally, we comment on the notion of universality. The first issue concerns the dependence of the fix point structure of the theory on the choice of coarse graining map. The coarse graining maps that we took into account all were based on injections of finite resolution smearing functions into the continuum, subject to the conditions (3.3) and (3.11) and thus are of a particular class. One would like to know, how much the fixed point structure depends on the particulars of the choice within this class. The second issue concerns the dependence of the fixed point structure on the initial family $\mu_{T, N, R, M}^{(0)}$. In $[58,59]$ we study both dependencies for the case of the Klein-Gordon field. To finish this section, let us mention that what has been said here can be generalised. Instead of labelling finite resolution smearing functions by integers $M, N \in \mathbb{N}_{0}^{2}$ we could use any partially ordered set $\mathcal{I}$ which can be partitioned into directed subsets. Here partially ordered means that there is a relation (symmetric, reflexive, transitive) $i<j$ between certain but not necessarily all elements $i, j \in \mathcal{I}$ and a subset $\mathcal{J} \subset \mathcal{I}$ is said to be directed if for any $i, j \in \mathcal{J}$ there exists $k \in \mathcal{J}$ such that $i, j<k$. In our case we say that $(M, N)<\left(M^{\prime}, N^{\prime}\right)$ if $M^{\prime}=2^{m} M, N^{\prime}=2^{n} N$ for some $(m, n) \in \mathbb{N}_{0}^{2}$ and thus the directed subsets of $\mathcal{I}$ are of the form $\mathcal{J}_{M, N}=\left\{\left(2^{m} M, 2^{n} N\right), m, n \in \mathbb{N}_{0}^{2}\right\}$ where $M, N$ are odd, positive integers. Evidently these sets are mutually disjoint and partition $\mathcal{I}$.

Dropping the labels $R, T$ for convenience we would then have for function spaces $L, L_{j}$ corresponding injections $I_{j}$, evaluations $E_{j}$, coarse grainings $I_{i j}=E_{j} \circ I_{i}$ for $i<j$ in the same directed subset subject to $I_{i i}=\mathrm{id}_{L_{i}}$ and $I_{j} \circ I_{i j}=I_{i}$. From here on, everything stays the same. Such a more abstract viewpoint is convenient, if one would like to define more general coarse graining transformations.

\section{Hamiltonian renormalisation}

In this section we intend to define Hamiltonian renormalisation in such a way, that the following diagram closes: Given a family of discretisations of the OS Data $\left(\mathcal{H}_{R, M}^{(0)}, \Omega_{R, M}^{(0)}, H_{R, M}^{(0)}\right)$ labelled by $M$ one can construct the corresponding Wiener measure family $\mu_{R, M}^{(0)}$. When restricted to smearing functions of time support $T$ and finite time resolution labelled by $N$ one obtains its temporal cylindrical projections $\mu_{T, N, R, M}$ family. We notice that while we can make contact to the previous section, the discretised measures that we obtain are of a special type: They are already cylindrically consistent with respect to coarse grainings in the time direction. The special role of time cannot be avoided in the Hamiltonian setting because the Hamiltonian generator of a contraction semi-group needs a continuous parameter and cannot be derived using a temporal lattice. Accordingly, we drop the label $T$ for the rest of this section assuming that the purely temporal renormalisation was already carried out. 
One can now perform path integral renormalisation as outlined in the previous section and obtain a fix point measure $\mu_{R}$. We will see that this measure, if it exists, is automatically reflection positive if the initial family is. From it we then obtain the continuum OS data $\left(\mathcal{H}_{R}, \Omega_{R}, H_{R}\right)$ by OS reconstruction. What we intend to find out is how to pass from the starting point $\left(\mathcal{H}_{R, M}^{(0)}, \Omega_{R, M}^{(0)}, H_{R, M}^{(0)}\right)$ directly to the same endpoint to $\left(\mathcal{H}_{R}, \Omega_{R}, H_{R}\right)$. In other words, the direct renormalisation from $\left(\mathcal{H}_{R, M}^{(0)}, \Omega_{R, M}^{(0)}, H_{R, M}^{(0)}\right)$ to $\left(\mathcal{H}_{R}, \Omega_{R}, H_{R}\right)$ allows us to define the corresponding Wiener measure $\mu_{R}$ and that measure coincides with the one that one obtains via path integral renormalisation.

The advantage of such a scheme, if it exists, is quite obvious: The construction of the complicated Wiener measures can be avoided altogether and one can stay entirely within the Hamiltonian setting. The disadvantage maybe is, that three ingredients must be renormalised instead of only one: The Hilbert space, the vacuum and the Hamiltonian annihilating that vacuum. By contrast, path integral renormalisation only needs to renormalise the measure.

Unfortunately, we will only be partially successful in doing so: While we are able to find an exact Hamiltonian renormalisation scheme that is equivalent to the path integral renormalisation scheme, this scheme still makes non-trivial reference to the Wiener measures at each renormalisation step. That is, while the Wiener measure is a derived concept fully determined by OS data, one still needs to construct it in an intermediate step. However, the derived renormalisation scheme motivates a different scheme which does stay entirely within the Hamiltonian framework without the need to construct the Wiener measure. Of course, there is no guarantee that the two schemes have the same fixed points. Nevertheless, for the second scheme, if it does have fixed points, these do define again Wiener measures in the continuum satisfying reflection positivity. Even for free field theories the renormalisation trajectories of both schemes are different, however their fixed points turn out to be identical.

The general setting is as follows:

From a given family of OS data $\left(\mathcal{H}_{R, M}^{(0)}, \Omega_{R, M}^{(0)}, H_{R, M}^{(0)}\right)$ let us construct the Wiener measure family $\mu_{R, M}^{(0)}$. That is, for a smearing function $F_{R, M}(\beta)=\sum_{k=1}^{n} f_{R, M}^{k} \delta\left(\beta, \beta_{k}\right)$ of discrete and finite time support $\beta_{1}<. .<\beta_{n}$ and finite spatial resolution

$\mu_{R, M}^{(0)}\left(W_{R, M}\left[\hat{F}_{R, M}\right]\right)=\left\langle\Omega_{R, M}^{(0)}, w_{R, M}\left[f_{R, M}^{n}\right] e^{\left.-\left(\beta_{n}-\beta_{n-1}\right) H_{R, M}^{(0)} \ldots e^{-\left(\beta_{2}-\beta_{1}\right) H_{R, M}^{(0)}} w_{R, M}\left[f_{R, M}^{1}\right] \Omega_{R, M}^{(0)}\right\rangle_{\mathcal{H}_{R, M}^{(0)}}}\right.$

As the measure is already temporally cylindrically consistent, we restrict the injection and evaluation maps of the previous section to the spatial variables. Thus, we have function spaces $L_{R}, L_{R, M}$ and the maps

$$
I_{R, M}: L_{R, M} \rightarrow L_{R} ; E_{R, M}: L_{R} \rightarrow L_{R, M}
$$

subject to the constraint

$$
E_{R, M} \circ I_{R, M}=\mathrm{id}_{L_{R, M}}
$$

from which one obtains the coarse graining maps

$$
I_{R, M \rightarrow 2^{m} M}=E_{R, 2^{m} M} \circ I_{R, M}
$$

subject to the constraints

$$
I_{R, 2^{m} M} \circ I_{R, M \rightarrow 2^{m} M}=I_{R, M}
$$

These maps are related to the ones for path integral renormalisation including the time direction by the assumption that the latter factorises into maps for the time direction and the above maps for the spatial direction, see (3.1). Then one carries out first only temporal renormalisation and arrives at the purely temporal fixed point $\mu_{R, M}^{(0)}$ for each spatial cut-off $M$ which are equivalent to the above OS data. 
In the next subsection we derive first the Hamiltonian renormalisation scheme which is indeed equivalent to the path integral renormalisation scheme. We call it the "Path Integral Induced Hamiltonian Renormalisation Scheme". This motivates the second subsection in which we define another renormalisation scheme which we coin "Direct Hamiltonian Renormalisation Scheme".

\subsection{Derivation of the Path Integral Induced Hamiltonian Renormalisation Scheme}

We begin by showing that the path integral renormalisation based on the coarse graining maps $I_{R, M \rightarrow 2 M}$ does not leave the space of families of time translation invariant, reflection invariant, reflection positive and reflection continuous measures if the initial family $\left\{\mu_{R, M}^{(0)}\right\}_{M \in \mathbb{N}}$ is of this kind.

Recall that path integral renormalisation is defined by a sequence $n \mapsto \mu_{R, M}^{(n)}$ of families $\left\{\mu_{R, M}^{(n)}\right\}_{M \in \mathbb{N}}$ defined recursively by

$$
\mu_{R, M}^{(n+1)}\left(W_{R, M}\left[F_{R, M}\right]\right):=\mu_{R, 2 M}^{(n)}\left(W_{R, 2 M}\left(I_{R, M \rightarrow 2 M} \cdot F_{R, M}\right]\right)
$$

where $I_{R, M \rightarrow 2 M}$ acts simultaneously at each time step only on the spatial arguments of $F_{R, M}$ and thus commutes with time reflection $\theta$ and time translation $T_{s}$.

Time-Translation-Invariance:

$$
\begin{aligned}
\mu_{R, M}^{(n+1)}\left(\mathcal{U}(s) \cdot W_{R, M}\left[F_{R, M}\right]\right) & =\mu_{R, M}^{(n+1)}\left(W_{R, M}\left[T_{s} \cdot F_{R, M}\right]\right) \\
& =\mu_{R, 2 M}^{(n)}\left(W_{R, 2 M}\left[I_{R, M \rightarrow 2 M} \cdot T_{s} \cdot F_{R, M}\right]\right) \\
& =\mu_{R, 2 M}^{(n)}\left(W_{R, 2 M}\left[T_{s} \cdot I_{R, M \rightarrow 2 M} \cdot F_{R, M}\right]\right) \\
& =\mu_{R, 2 M}^{(n)}\left(\mathcal{U}(s) \cdot W_{R, 2 M}\left[I_{R, M \rightarrow 2 M} \cdot F_{R, M}\right]\right) \\
& =\mu_{R, 2 M}^{(n)}\left(W_{R, 2 M}\left[I_{R, M \rightarrow 2 M} \cdot F_{R, M}\right]\right) \\
& =\mu_{R, M}^{(n+1)}\left(W_{R, M}\left[F_{R, M}\right]\right)
\end{aligned}
$$

Time-Reflection-Invariance:

$$
\begin{aligned}
\mu_{R, M}^{(n+1)}\left(R \cdot W_{R, M}\left[F_{R, M}\right]\right) & =\mu_{R, M}^{(n+1)}\left(W_{R, M}\left[\theta \cdot F_{R, M}\right]\right) \\
& =\mu_{R, 2 M}^{(n)}\left(W_{R, 2 M}\left[I_{R, M \rightarrow 2 M} \cdot \theta \cdot F_{R, M}\right]\right) \\
& =\mu_{R, 2 M}^{(n)}\left(W_{R, 2 M}\left[\theta \cdot I_{R, M \rightarrow 2 M} \cdot F_{R, M}\right]\right) \\
& =\mu_{R, 2 M}^{(n)}\left(R \cdot W_{R, 2 M}\left[I_{R, M \rightarrow 2 M} \cdot F_{R, M}\right]\right) \\
& =\mu_{R, 2 M}^{(n)}\left(W_{R, 2 M}\left[I_{R, M \rightarrow 2 M} \cdot F_{R, M}\right]\right) \\
& =\mu_{R, M}^{(n+1)}\left(W_{R, M}\left[F_{R, M}\right]\right)
\end{aligned}
$$

Reflection Positivity:

$$
\begin{aligned}
& \mu_{R, M}^{(n+1)}\left(W_{R, M}\left[F_{R, M}\right]^{*}\left(R \cdot W_{R, M}\left[F_{R, M}\right]\right)\right)=\sum_{\alpha} z_{\alpha} \mu_{R, M}^{(n+1)}\left(W_{R, M}\left[F_{\alpha, R}\left(F_{R, M}^{*}, \theta \cdot F_{R, M}\right)\right]\right) \\
= & \sum_{\alpha} z_{\alpha} \mu_{R, 2 M}^{(n)}\left(W_{R, 2 M}\left[I_{R, M \rightarrow 2 M} \cdot F_{\alpha, R}\left(F_{R, M}^{*}, \theta \cdot F_{R, M}\right)\right]\right) \\
= & \sum_{\alpha} z_{\alpha} \mu_{R, 2 M}^{(n)}\left(W_{R, 2 M}\left[F_{\alpha, R}\left(\left(I_{R, M \rightarrow 2 M} \cdot F_{R, M}\right)^{*}, \theta \cdot I_{R, M \rightarrow 2 M} \cdot F_{R, M}\right)\right]\right) \\
= & \mu_{R, 2 M}^{(n)}\left(\left(W_{R, 2 M}\left[I_{R, M \rightarrow 2 M} \cdot F_{R, M}\right]\right)^{*}\left(R \cdot W_{R, M}\left[I_{R, M \rightarrow 2 M} \cdot F_{R, M}\right]\right)\right) \\
\geq & 0
\end{aligned}
$$

Here we actually treated only a special case, namely that we just have a single function $F_{R, M}$ with a single time dependence. However, the general case is completely analogous and just requires the 
Weyl element decomposition at each coinciding point of time and will be left to the reader. We have assumed the equivariance of the Weyl algebra relations (2.2) and thus its spacetime equivalent with respect to the coarse graining maps, that is

$$
I_{R, M \rightarrow 2 M} \cdot F_{R, M}^{*}=\left(I_{R, M \rightarrow 2 M} \cdot F_{R, M}\right)^{*}, I_{R, M \rightarrow 2 M} \cdot F_{\alpha, R}\left(F_{R, M}, F_{R, M}^{\prime}\right)=F_{\alpha, R}\left(I_{R, M \rightarrow 2 M} \cdot F_{R, M}, I_{R, M \rightarrow 2 M} \cdot F_{R, M}^{\prime}\right)
$$

which is satisfied in all known examples. Note that the Weyl relations (2.2) of the continuum induce the same relations on the finite resolution analogues due to

$$
W_{R}\left[I_{R, M} \cdot F_{R, M}\right]=: W_{R, M}\left[F_{R, M}\right]
$$

in particular the constants $z_{\alpha}$ and the composition functions $F_{\alpha}$ do not depend on $M$. That reflection continuity (or equivalently time translation continuity) is also inherited is obvious since again time translations commute with the coarse graining map and thus the corresponding semigroups have a generator.

It follows that each of the measures $\mu_{R, M}^{(n)}$ defines OS data $\left(\mathcal{H}_{R, M}^{(n)}, \Omega_{R, M}^{(n)}, H_{R, M}^{n)}\right)$ by OS reconstruction and we now want to describe them in more detail.

Let $\left[W_{R, M}\left[F_{R, M}\right]\right]_{\mu_{R, M}^{(n)}} \in \mathcal{H}_{R, M}^{(n)}$ denote the equivalence class of the vector $W_{R, M}\left[F_{R, M}\right]$, T$\operatorname{supp}\left(F_{R, M}\right) \subset \mathbb{R}_{+}$with respect to the reflection positive inner product defined by $\mu_{R, M}^{(n)}$, in particular $\Omega_{R, M}^{(n)}=[1]_{\mu_{R, M}^{(n)}}$. Let

$$
J_{R, M \rightarrow 2 M}^{(n)}: \mathcal{H}_{R, M}^{(n+1)} \rightarrow \mathcal{H}_{R, 2 M}^{(n)} ; \quad\left[W_{R, M}\left[F_{R, M}\right]\right]_{\mu_{R, M}^{(n+1)}} \mapsto\left[W_{R, 2 M}\left[I_{R, M \rightarrow 2 M} \cdot F_{R, M}\right]\right]_{\mu_{R, 2 M}^{(n)}}
$$

This defines a sequence of densely defined families of embeddings of Hilbert spaces. Then by the definition of the reflection positive inner product and the assumed Weyl algebra relations by a similar calculation as in (4.9)

$$
\begin{aligned}
& \left\langle\left[W_{R, M}\left[F_{R, M}\right]\right]_{\mu_{R, M}^{(n+1)}},\left[W_{R, M}\left[F_{R, M}^{\prime}\right]\right]_{\mu_{R, M}^{(n+1)}}\right\rangle_{\mathcal{H}_{R, M}^{(n+1)}}=\mu_{R, M}^{(n+1)}\left(\left(W_{R, M}\left[F_{R, M}\right]\right)^{*} W_{R, M}\left[\theta \cdot F_{R, M}^{\prime}\right]\right) \\
= & \mu_{R, 2 M}^{(n)}\left(\left(W_{R, 2 M}\left[I_{R, M \rightarrow 2 M} \cdot F_{R, M}\right]\right)^{*} W_{R, 2 M}\left[\theta \cdot I_{R, M \rightarrow 2 M} \cdot F_{R, M}^{\prime}\right]\right) \\
= & \left\langle\left[W_{R, 2 M}\left[I_{R, M \rightarrow 2 M} \cdot F_{R, M}\right]\right]_{\mu_{R, 2 M}^{(n)}},\left[W_{R, 2 M}\left[I_{R, M \rightarrow 2 M} \cdot F_{R, M}^{\prime}\right]\right]_{\mu_{R, 2 M}^{(n)}}\right\rangle_{\mathcal{H}_{R, 2 M}^{(n)}} \\
= & \left\langle J_{R, M}^{(n)}\left[W_{R, M}\left[F_{R, M}\right]\right]_{\mu_{R, M}^{(n+1)}}, J_{R, M}^{(n)}\left[W_{R, M}\left[F_{R, M}^{\prime}\right]\right]_{\mu_{R, M}^{(n+1)}}\right\rangle_{\mathcal{H}_{R, 2 M}^{(n)}}
\end{aligned}
$$

It follows that for each $n$ the family of maps $J_{R, M \rightarrow 2 M}^{(n)}$ defines a family of isometric embeddings $\mathcal{H}_{R, M}^{(n+1)} \rightarrow \mathcal{H}_{R, 2 M}^{(n)}$, i.e.

$$
\left(J_{R, M \rightarrow 2 M}^{(n)}\right)^{\dagger} J_{R, M \rightarrow 2 M}^{(n)}=1_{\mathcal{H}_{R, M}^{(n+1)}}
$$

It follows that

$$
P_{R, M \rightarrow 2 M}^{(n)}:=J_{R, M \rightarrow 2 M}^{(n)}\left(J_{R, M \rightarrow 2 M}^{(n)}\right)^{\dagger}
$$

are projections onto the subspace $J_{R, M \rightarrow 2 M}^{(n)} \mathcal{H}_{R, M}^{(n+1)}$ of $\mathcal{H}_{R, 2 M}^{(n)}$, that is

$$
\left(P_{R, M \rightarrow 2 M}^{(n)}\right)^{2}=J_{R, M \rightarrow 2 M}^{(n)}\left\{\left(J_{R, M \rightarrow 2 M}^{(n)}\right)^{\dagger} J_{R, M \rightarrow 2 M}^{(n)}\right\}\left(J_{R, M \rightarrow 2 M}^{(n)}\right)^{\dagger}=P_{R, M \rightarrow 2 M}^{(n)}
$$

For later reference we also note the identities

$$
P_{R, M \rightarrow 2 M}^{(n)} J_{R, M \rightarrow 2 M}^{(n)}=J_{R, M \rightarrow 2 M}^{(n)} \Rightarrow\left(J_{R, M \rightarrow 2 M}^{(n)}\right)^{\dagger} P_{R, M \rightarrow 2 M}^{(n)}=\left(J_{R, M \rightarrow 2 M}^{(n)}\right)^{\dagger}
$$


Next we find for the flow of the Hamiltonians by the already familiar calculations

$$
\begin{aligned}
& \left\langle\left[W_{R, M}\left[F_{R, M}\right]\right]_{\mu_{R, M}^{(n+1)}}, e^{-\beta H_{R, M}^{(n+1)}}\left[W_{R, M}\left[F_{R, M}^{\prime}\right]\right]_{\mu_{R, M}^{(n+1)}}\right\rangle_{\mathcal{H}_{R, M}^{(n+1)}} \\
= & \mu_{R, M}^{(n+1)}\left(\left(W_{R, M}\left[F_{R, M}\right]\right)^{*} W_{R, M}\left[\theta \cdot T_{\beta} \cdot F_{R, M}^{\prime}\right]\right) \\
= & \mu_{R, 2 M}^{(n)}\left(\left(W_{R, 2 M}\left[I_{R, M \rightarrow 2 M} \cdot F_{R, M}\right]\right)^{*} W_{R, 2 M}\left[I_{R, M \rightarrow 2 M} \cdot \theta \cdot T_{\beta} \cdot F_{R, M}^{\prime}\right]\right) \\
= & \mu_{R, 2 M}^{(n)}\left(\left(W_{R, 2 M}\left[I_{R, M \rightarrow 2 M} \cdot F_{R, M}\right]\right)^{*} W_{R, 2 M}\left[\theta \cdot T_{\beta} \cdot I_{R, M \rightarrow 2 M} \cdot F_{R, M}^{\prime}\right]\right) \\
= & \left\langle\left[W_{R, 2 M}\left[I_{R, M \rightarrow 2 M} \cdot F_{R, M}\right]\right]_{\mu_{R, 2 M}^{(n)}}, e^{\left.-\beta H_{R, 2 M}^{(n)}\left[W_{R, 2 M}\left[I_{R, M \rightarrow 2 M} \cdot F_{R, M}^{\prime}\right]\right]_{\mu_{R, 2 M}^{(n)}}\right\rangle_{\mathcal{H}_{R, 2 M}^{(n)}}}\right. \\
= & \left\langle J_{R, M \rightarrow 2 M}^{(n)}\left[W_{R, M}\left[F_{R, M}\right]\right]_{\mu_{R, 2 M}^{(n+1)}}, e^{-\beta H_{R, 2 M}^{(n)}} J_{R, M \rightarrow 2 M}^{(n)}\left[W_{R, M}\left[F_{R, M}^{\prime}\right]\right]_{\left.\mu_{R, M}^{(n+1)}\right\rangle_{\mathcal{H}_{R, 2 M}^{(n)}}}(4.18\right.
\end{aligned}
$$

whence for all $\beta>0$

$$
e^{-\beta H_{R, M}^{(n+1)}}=\left(J_{R, M \rightarrow 2 M}^{(n)}\right)^{\dagger} e^{-\beta H_{R, 2 M}^{(n)}} J_{R, M \rightarrow 2 M}^{(n)}
$$

while for $\beta=0$ we recover the isometry condition (4.14).

Taking the $l$-th derivative ${ }^{4}$ of (4.19) at $\beta=0$ (which is possible on a dense and invariant domain of the Hamiltonian (analytic vectors) due to the preservation of the OS continuity during renormalisation) we find

$$
\left(H_{R, M}^{(n+1)}\right)^{l}=\left(J_{R, M \rightarrow 2 M}^{(n)}\right)^{\dagger}\left(H_{R, 2 M}^{(n)}\right)^{l} J_{R, M \rightarrow 2 M}^{(n)}
$$

For $l=1$ we conclude that the sequence of families of Hamiltonians remains symmetric if the initial family is. Combining (4.20) for $l=1,2$ we find

$$
\left(J_{R, M \rightarrow 2 M}^{(n)}\right)^{\dagger}\left(H_{R, 2 M}^{(n)}\right)^{2} J_{R, M \rightarrow 2 M}^{(n)}=\left(J_{R, M \rightarrow 2 M}^{(n)}\right)^{\dagger} H_{R, 2 M}^{(n)} P_{R, M \rightarrow 2 M}^{(n)} H_{R, 2 M}^{(n)} J_{R, M \rightarrow 2 M}^{(n)}
$$

Multiplying (4.21) with $\left(J_{R, M \rightarrow 2 M}^{(n)}\right)^{\dagger}, J_{R, M \rightarrow 2 M}^{(n)}$ from the right and left respectively yields the identity

$$
P_{R, M \rightarrow 2 M}^{(n)}\left(H_{R, 2 M}^{(n)}\right)^{2} P_{R, M \rightarrow 2 M}^{(n)}=P_{R, M \rightarrow 2 M}^{(n)} H_{R, 2 M}^{(n)} P_{R, M \rightarrow 2 M}^{(n)} H_{R, 2 M}^{(n)} P_{R, M \rightarrow 2 M}^{(n)}
$$

which is equivalent to

$$
\begin{aligned}
0 & =P_{R, M \rightarrow 2 M}^{(n)} H_{R, 2 M}^{(n)}\left[1_{\mathcal{H}_{R, 2 M}^{(n)}}-P_{R, M \rightarrow 2 M}^{(n)}\right] H_{R, 2 M}^{(n)} P_{R, M \rightarrow 2 M}^{(n)} \\
& =P_{R, M \rightarrow 2 M}^{(n)} H_{R, 2 M}^{(n)}\left[P_{R, M \rightarrow 2 M}^{(n)}\right]_{\perp} H_{R, 2 M}^{(n)} P_{R, M \rightarrow 2 M}^{(n)} \\
& =\left(P_{R, M \rightarrow 2 M}^{(n)} H_{R, 2 M}^{(n)}\left[P_{R, M \rightarrow 2 M}^{(n)}\right]_{\perp}\right)\left(P_{R, M \rightarrow 2 M}^{(n)} H_{R, 2 M}^{(n)}\left[P_{R, M \rightarrow 2 M}^{(n)}\right]_{\perp}\right)^{\dagger}=: A^{\dagger} A
\end{aligned}
$$

It follows $\left\langle\Psi_{R, 2 M}, A^{\dagger} A \Psi_{R, 2 M}\right\rangle=\left\|A \Psi_{R, 2 M}\right\|^{2}=0$ for all $\Psi_{R, 2 M}$ in the domain of $A$ so that $A=0$. It follows

$H_{R, 2 M}^{(n)} P_{R, M \rightarrow 2 M}^{(n)}=P_{R, M \rightarrow 2 M}^{(n)} H_{R, 2 M}^{(n)} P_{R, M \rightarrow 2 M}^{(n)}=\left(P_{R, M \rightarrow 2 M}^{(n)} H_{R, 2 M}^{(n)} P_{R, M \rightarrow 2 M}^{(n)}\right)^{\dagger}=P_{R, M \rightarrow 2 M}^{(n)} H_{R, 2 M}^{(n)}$

so that $H_{R, 2 M}^{(n)}$ has the remarkable property to preserve the subspace $P_{R, M \rightarrow 2 M}^{(n)} \mathcal{H}_{R, 2 M}^{(n)}$. This explains why (4.19) is consistent with the semigroup property. We note that from the semigroup property

$$
\begin{aligned}
e^{-\left(\beta_{1}+\beta_{2}\right) H_{R, M}^{(n+1)}} & =\left(J_{R, M \rightarrow 2 M}^{(n)}\right)^{\dagger} e^{-\left(\beta_{1}+\beta_{2}\right) H_{R, 2 M}^{(n)}} J_{R, M \rightarrow 2 M}^{(n)} \\
=e^{-\beta_{1} H_{R, M}^{(n+1)}} e^{-\beta_{2} H_{R, M}^{(n+1)}} & =\left(J_{R, M \rightarrow 2 M}^{(n)}\right)^{\dagger} e^{-\beta_{1} H_{R, 2 M}^{(n)}} P_{R, M \rightarrow 2 M}^{(n)} e^{-\beta_{2} H_{R, 2 M}^{(n)}} J_{R, M \rightarrow 2 M}^{(n)}
\end{aligned}
$$

\footnotetext{
${ }^{4}$ This makes sense on analytic vectors that exist owing to the granted self-adjointness of the Hamiltonians. If one wants to avoid domain questions, then one could carry out the same analysis that follows from here on and would conclude that $\left[P_{R, M \rightarrow 2 M}^{(n)}, e^{-\beta H_{R, 2 M}^{(n)}}\right]=0$ for all $\beta$. Thus all spectral projections of $H_{R, 2 M}^{(n)}$ commute with $P_{R, 2 M}^{(n)}$ which is exactly what is meant when talking about the commutativity of unbounded operators.
} 
This property is at first sight indeed astonishing and in fact counter intuitive: Suppose that for all $n \in \mathbb{N}$ a representative of $\left[W_{R, M}\left[F_{R, M}\right]\right]_{\mu_{R, M}^{(n)}}$ is given by a linear combination of vectors of the form $w_{R, M}\left[f_{R, M}\right] \Omega_{R, M}^{(n)}$ for suitable $f_{R, M}$ with say $\Omega_{R, M}^{(n)}=1, \forall M, n$ in the ground state representation, i.e. in terms of sharp time zero fields on a lattice labelled by $M$. Then $J_{R, M \rightarrow 2 M}^{(n)}$ is actually independent of $n$ and basically embeds those vectors into the lattice labelled by $2 M$ using the map $I_{R, M \rightarrow 2 M}$ e.g. by associating a constant value to the smearing fields in a block within the fine lattice that corresponds to a point in the coarse lattice. Then (4.24) would imply that the Hamiltonian on the fine lattice actually preserves the space of vectors with smearing functions of that special type and that property is certainly violated in all applications.

The apparent contradiction is resolved by noticing that there is no guarantee that $\left[W_{R, M}\left[F_{R, M}\right]\right]_{\mu_{R, M}^{(n)}}$ can in fact be represented by vectors in the linear span of the $w_{R, M}\left[f_{R, M}\right] \Omega_{R, M}^{(n)}$. This is because of the innocent looking dependence of the equivalence class $[\cdot]_{\mu_{R, M}^{(n)}}$ on the measure. Even if the initial equivalence class can be represented in terms of sharp time zero fields of a single field species, this may no longer be the case after already one renormalisation step. Indeed, this is what happens already in the free field theory case. There one can label $\left[W_{R, M}\left[F_{R, M}\right]\right]_{\mu_{R, M}^{(n)}}$ either as a single field species at an increasing number of sharp times (as $n \rightarrow \infty)$ or as a single field at single sharp time zero which is composed of an increasing number of mutually interacting free field species. Thus the intuition that one may have with the meaning of $J_{R, M \rightarrow 2 M}^{(n)}$ is quite misleading.

For the same reason, the quite compactly looking condition (4.20) for $l=1$ only partly fulfils our aim to directly compute the flow of the Hamiltonians purely within the Hamiltonian theory: Starting with initial OS data $\left(\mathcal{H}_{R, M}^{(0)}, \Omega_{R, M}^{(0)}, H_{R, M}^{(0)}\right)$ we have to compute the corresponding Wiener measures $\mu_{R, M}^{(0)}$ in order to define $J_{R, M \rightarrow 2 M}^{(0)}$ via (4.12) As alluded to above, this may not only drastically change the meaning of $w_{R, M}\left[f_{R, M}\right]$ if we want to keep a sharp zero-time interpretation of the equivalence classes, we also cannot avoid the computation of the Wiener measure as we actually intended to do.

We close this subsection with the remark that the path integral induced Hamiltonian renormalisation discussed above bears a certain similarity with the Hamiltonian renormalisation scheme advocated in $[41,42]$ in the sense that both schemes attempt to push the renormalisation flow of the Hamiltonian into a block diagonal form. The difference is that, in those latter schemes in contrast to what happens here, first this is done for two fixed resolutions (the high energy cut-off and the low energy effective resolution) and not for a whole family thereof and second without changing the "size" and the vacuum of the Hilbert space at the same resolution. A better understanding of the relation between these two schemes and the one we will propose in the next section is desirable and will be subject of a future publication.

\subsection{Motivation of the Direct Hamiltonian Renormalisation Scheme}

The caveat to avoiding the computation of the Wiener measure pointed out in the previous subsection and the complications associated with the changing realisation of the equivalence classes of vectors under the path integral renormalisation flow suggest a natural more direct Hamiltonian renormalisation scheme which stays maximally close to the path integral renormalisation scheme:

Starting from initial OS data $\left(\mathcal{H}_{R, M}^{(0)}, \Omega_{R, M}^{(0)}, H_{R, M}^{(0)}\right)$ we define the maps

$$
j_{R, M \rightarrow 2 M}^{(n)}: \mathcal{H}_{R, M}^{(n+1)} \rightarrow \mathcal{H}_{R, 2 M}^{(n)} ; w_{R, M}\left[f_{R, M}\right] \Omega_{R, M}^{(n+1)} \mapsto w_{R, 2 M}\left[I_{R, M \rightarrow 2 M} \cdot f_{R, M}\right] \Omega_{R, 2 M}^{(n)}
$$

in terms of Weyl elements of single species sharp time zero fields which are implicitly assumed to span $\mathcal{H}_{R, M}^{(n)}$ for all $n$. In particular for $f_{R, M}=0$

$$
j_{R, M \rightarrow 2 M}^{(n)} \Omega_{R, M}^{(n+1)}=\Omega_{R, 2 M}^{(n)}
$$


Definition (4.26) replaces definition (4.12). Note that the actual action of (4.26), in contrast to $J_{R, M}^{(n)}$ does not depend on $n$ and is purely geometrically defined entirely in terms of $I_{R, M \rightarrow 2 M}$, except for (4.27). In the ground state representation, however, $\Omega_{R, M}^{(n)}=1$ for all $n, M$.

Next we insist on the analogue of the isometry condition (4.14)

$$
\left(j_{R, M \rightarrow 2 M}^{(n)}\right)^{\dagger} j_{R, M \rightarrow 2 M}^{(n)}=1_{\mathcal{H}_{R, M}^{(n+1)}}
$$

which in practical terms leads to a flow of Hilbert space measures (recall that

$\left.\nu_{R, M}()=.\left\langle\Omega_{R, M}, . \Omega_{R, M}\right\rangle_{\mathcal{H}_{R, M}}\right)$

$$
\nu_{R, M}^{(n+1)}\left(w_{R, M}\left[f_{R, M}\right]\right)=\nu_{R, 2 M}^{(n)}\left(w_{R, 2 M}\left[I_{R, M \rightarrow 2 M} \cdot f_{R, M}\right]\right)
$$

Finally, we impose the analogue of (4.20) for $l=1$

$$
H_{R, M}^{(n+1)}:=\left(j_{R, M \rightarrow 2 M}^{(n)}\right)^{\dagger} H_{R, M}^{(n)} j_{R, M \rightarrow 2 M}^{(n)}
$$

but not the analogue of the stronger condition (4.19) since it would imply $\left[H_{R, 2 M}^{(n)}, p_{R, M \rightarrow 2 M}^{(n)}\right]=0$ for the projector $p_{R, M \rightarrow 2 M}^{(n)}=j_{R, M \rightarrow 2 M}^{(n)}\left(j_{R, M \rightarrow 2 M}^{(n)}\right)^{\dagger}$ which is not physically viable as we have argued above.

Note that the flow of Hamiltonians and vacua is consistent since

$$
H_{R, M}^{(n+1)} \Omega_{R, M}^{(n+1)}=\left(j_{R, M \rightarrow 2 M}^{(n)}\right)^{\dagger} H_{R, 2 M}^{(n)}\left[j_{R, M \rightarrow 2 M}^{(n)} \Omega_{R, M}^{(n+1)}\right]=\left(j_{R, M \rightarrow 2 M}^{(n)}\right)^{\dagger} H_{R, 2 M}^{(n)} \Omega_{R, 2 M}^{(n)}=0
$$

Conditions (4.26), (4.28) and (4.30) define a flow of OS data or equivalently Wiener measures which is in close analogy to the conditions (4.14), (4.12) and (4.20) stated in the previous subsection. However, that flow will generically not coincide with the flow defined in the previous section, already not for the free field. However, we are not interested in the renormalisation trajectory but rather in its fixed points, i.e. we want to know whether the direct and the path integral Hamiltonian flow lead to the same fixed points. At least for the free field theory case the answer is in the affirmative and the way this happens is quite interesting: Namely, while the direct Hamiltonian flow always stays within a single field species context, the path integral flow develops an infinite number of field species with specific coupling "constants", the non-vanishing number of which increase with each renormalisation step. These constants play a role similar to the ones of the Källen-Lehmann spectral function of a generalised free field. However, all but a single member of these fields develop an infinite mass in the continuum limit $M \rightarrow \infty$ and thus decouple. The remaining single field of finite mass of the path integral flow can be identified with the one of the direct Hamiltonian flow in the continuum limit. For this reason, both schemes result in the same continuum theory which of course coincides with the free field of a single finite mass.

What can be said about the fixed points of the direct Hamiltonian flow when they exist? Let us denote the fixed point structures by $j_{R, M \rightarrow 2 M}, \mathcal{H}_{R, M}, \Omega_{R, M}, H_{R, M}$. Then

$$
j_{R, M \rightarrow 2^{n} M}:=j_{R, 2^{n-1} M \rightarrow 2^{n} M} \cdot . . \cdot j_{R, M \rightarrow 2 M}: \mathcal{H}_{R, M} \rightarrow \mathcal{H}_{R, 2^{n} M}
$$

defines a family of isometric injections of Hilbert spaces labelled by the partially ordered set $M:=$ $(k, n):=(2 k+1) 2^{n}, k, n \in \mathbb{N}_{0}$ where $M<M^{\prime}$ iff $k=k^{\prime}$ and $n<n^{\prime}$. It has directed (in fact linearly ordered) subsets, namely those for fixed $k$. The injections satisfy the consistency condition

$$
j_{R, M_{2} \rightarrow M_{3}} \cdot j_{R, M_{1} \rightarrow M_{2}}=j_{R, M_{1} \rightarrow M_{3}} \forall M_{1}<M_{2}<M_{3}
$$

and thus we can form the inductive limit Hilbert space $\mathcal{H}_{R}^{k}$ which always exists, see appendix $\mathrm{A}$ and induces isometric injections $j_{R, M}: \mathcal{H}_{R, M} \rightarrow \mathcal{H}_{R}^{k}, M=(k, n)$ satisfying the consistency condition

$$
j_{R, M^{\prime}} \cdot j_{R, M \rightarrow M^{\prime}}=j_{R, M} \quad \forall M<M^{\prime}
$$


Equivalently we may consider the partially ordered and directed family of measures $\nu_{R, M}=\left\langle\Omega_{R, M}, . \Omega_{R, M}\right\rangle_{\mathcal{H}_{R, M}}$ which by construction are cylindrically consistent

$$
\begin{aligned}
\nu_{R, M^{\prime}}\left(w_{R, M^{\prime}}\left[I_{R, M \rightarrow M^{\prime}} \cdot f_{R, M}\right]\right) & =\left\langle\Omega_{R, M^{\prime}}, w_{R, M^{\prime}}\left[I_{R, M \rightarrow M^{\prime}} \cdot f_{R, M}\right] \Omega_{R, M^{\prime}}\right\rangle_{\mathcal{H}_{R, M^{\prime}}} \\
& =\left\langle j_{R, M \rightarrow M^{\prime}} \Omega_{R, M}, j_{R, M \rightarrow M^{\prime}} w_{R, M}\left[f_{R, M}\right] \Omega_{R, M}\right\rangle_{\mathcal{H}_{R, M^{\prime}}} \\
& =\left\langle\Omega_{R, M}, w_{R, M}\left[f_{R, M}\right] \Omega_{R, M}\right\rangle_{\mathcal{H}_{R, M}} \\
& =\nu_{R, M}\left(w_{R, M}\left[f_{R, M}\right]\right)
\end{aligned}
$$

for all $M<M^{\prime}$. Thus, by suitable extension theorems [9] a $\sigma$-additive measure $\nu_{R}^{k}$ on the projective limit

$$
\begin{aligned}
\gamma_{R}^{k}=\left\{\left(\phi_{R, M}\right)_{M \in \mathbb{N}} ;\right. & \phi_{R, M^{\prime}}\left[I_{R, M \rightarrow M^{\prime}} \cdot f_{R, M}\right]=\phi_{R, M}\left[f_{R, M}\right] \\
\left.\forall M=(k, n), M^{\prime}=\left(k, n^{\prime}\right), n<n^{\prime}, \quad f_{R, M} \in L_{R, M}\right\} &
\end{aligned}
$$

exists and we have $\mathcal{H}_{R}^{k} \equiv L_{2}\left(\gamma_{R}^{k}, d \nu_{R}\right)$. We call the fixed point universal if $\mathcal{H}_{R}=\mathcal{H}_{R}^{k} \forall k \in \mathbb{N}$ is independent of $k$ which is an expected property because we associate $\mathcal{H}_{R}^{k}$ with the continuum limit $\lim _{n \rightarrow \infty} \mathcal{H}_{R, k 2^{n}}$ and that limit is expected to be independent of $k$. In fact, an even higher degree of universality is believed to occur: For instance, nothing is sacred about the factor of 2 in our coarse graining map $I_{R, M \rightarrow 2 M}$, we could have studied the flow for any $I_{R, M \rightarrow q M}, q \in \mathbb{N}, q>1$ and would expect independence of the inductive or projective limit of $q$. In principle we can even do this for all $M<M^{\prime}, M, M^{\prime} \in \mathbb{N}$ although then the computation of the flow becomes much more technically involved. These kinds of universality properties are studied in our companion paper [58].

Likewise, we have a family of Hamiltonian operators satisfying the condition

$$
j_{R, M \rightarrow M^{\prime}}^{\dagger} H_{R, M^{\prime}} j_{R, M \rightarrow M^{\prime}}=H_{R, M}
$$

for all $M<M^{\prime}$. This reminds of the structure of a partially ordered and directed system of densely defined operators $A_{M}$ with domains $D_{R, M}$ such that $j_{R, M \rightarrow M^{\prime}} D_{R, M} \subset D_{R, M^{\prime}}$ for which one has the condition for all $M<M^{\prime}$

$$
A_{M^{\prime}} \cdot j_{R, M \rightarrow M^{\prime}}=j_{R, M \rightarrow M^{\prime}} \cdot A_{M}
$$

see appendix $\mathrm{A}$. Such an inductive system of operators always has a densely defined inductive limit $A$ on the corresponding inductive limit Hilbert space satisfying $A j_{R, M}=j_{R, M} A_{M}$. However, (4.38) is much stronger than (4.37): Multiplying (4.38) from the left with $j_{R, M \rightarrow M^{\prime}}^{\dagger}$ we indeed obtain (4.37) due to isometry $j_{R, M \rightarrow M^{\prime}}^{\dagger} j_{R, M \rightarrow M^{\prime}}=1_{\mathcal{H}_{R M}}$ for $A_{M}=H_{R, M}$. However, multiplying (4.37) from the left with $j_{R, M \rightarrow M^{\prime}}$ only yields

$$
p_{R, M \rightarrow M^{\prime}} H_{R, M \rightarrow M^{\prime}} j_{R, M \rightarrow M^{\prime}}=j_{R, M \rightarrow M^{\prime}} H_{R, M}, \quad p_{R, M \rightarrow M^{\prime}}=j_{R, M \rightarrow M^{\prime}} j_{R, M \rightarrow M^{\prime}}^{\dagger}
$$

which yields (4.38) iff $\left[p_{R, M \rightarrow M^{\prime}}, H_{R, M^{\prime}}\right]=0$ since due to isometry $p_{R, M \rightarrow M^{\prime}} j_{R, M \rightarrow M^{\prime}}=j_{R, M \rightarrow M^{\prime}}$. Indeed, it would have been physically wrong if the renormalisation conditions would yield the structure of an inductive system of operators which always has an inductive limit $A$ because $A j_{R, M}=j_{R, M} A_{M}$ means that $A$ preserves all the subspaces $j_{R, M} \mathcal{H}_{R, M}$ of $\mathcal{H}_{R}$ separately while we would generically expect correlations (non-vanishing matrix elements) between arbitrary pairs of such subspaces. Moreover, we would have completely sidestepped Haag's theorem [71] which states that different Hamiltonians generically cannot be defined on the same Hilbert space. Indeed, all that we are granted to obtain, if a fixed point of OS data exists, is a quadratic form $H_{R}^{k}$ densely defined on the finite linear span of the vectors of the form $j_{R, M} \psi_{R, M}, \psi_{R, M} \in D_{R, M} \subset \mathcal{H}_{R, M}^{k}$ with $M=(k, n)$, denoted by $D_{R}^{k}$. On the other hand, it is maybe not completely hopeless that this quadratic form can be extended as a self-adjoint operator on $\mathcal{H}_{R}^{k}$ because $H_{R}^{k}$ is symmetric and positive. Namely, if it is in fact closable $e^{5}$ it has a unique Friedrichs extension as a self-adjoint positive operator [72]. This

\footnotetext{
${ }^{5}$ That is, $D_{R}^{k}$ can be extended to a complete space with respect to the norm $\|\cdot\|_{R}^{k}:=\sqrt{\left\langle., H_{R}^{k} \cdot\right\rangle_{\mathcal{H}_{R}^{k}}}$ and $H_{R}^{k}$ stays positive on that extension.
} 
hope is based on the fact that the renormalisation flow carefully keeps track of the vacua annihilated by the finite resolution Hamiltonians. We include it in our definition of universality if $H_{R}^{k}=H_{R}$ is independent of $k$ which is again plausible since we expect $H_{R}^{k}$ to be associated with $\lim _{n \rightarrow \infty} H_{R, k 2^{n}}$.

\section{Outlook: Hamiltonian Renormalisation of Generally Covari- ant Theories}

In this paper we exploited the 1-1 correspondence between (Euclidian) time translation invariant, time reflection invariant and reflection positive measures $\mu$ (OS measures) on the one hand and their corresponding OS data $(\mathcal{H}, \Omega, H)$ in order to motivate the definition of a direct Hamiltonian flow of the OS data. This correspondence uses the OS reconstruction and the Wiener measure construction algorithms respectively. On the path integral side, renormalisation involves the introduction of families of finite resolution measures $M \mapsto \mu_{R M}$ where $R$ is a fixed spatial IR cut-off and $M$ plays the role of an UV cut-off which labels the family. Upon picking a spatial block spin transformation also called a coarse graining map which preserves the space of OS measures, the path integral flow generates a sequence of measures $n \mapsto \mu_{R M}^{(n)}$ by defining the measure with parameters $(n+1, M)$ from the measure with parameters $(n, 2 M)$. By OS construction we may define the OS data $\left(\mathcal{H}_{R M}^{(n)}, \Omega_{R M}^{(n)}, H_{R M}^{(n)}\right)$ from $\mu_{R M}^{(n)}$. We showed how the OS data with parameters $(n+1, M)$ and $(n, 2 M)$ are related. This relation, if one wants to stay completely within the Hamiltonian setting, is unsatisfactory in the sense that one needs to construct the Wiener measure for the OS data with parameters $(n, 2 M)$ in an intermediate step, a complicated step which one would like to avoid. However, that relation suggests a closely related modified flow that directly relates the OS data with parameters $(n+1, M)$ and $(n, 2 M)$ respectively without recourse to the Wiener measure. This direct flow thus gives directly a sequence

$\left(\mathcal{H}_{R M}^{\prime(n)}, \Omega_{R M}^{\prime(n)}, H_{R M}^{\prime(n)}\right)$ of OS data from which one could construct a family of OS measures $\mu_{R M}^{\prime(n)}$ using the Wiener measure construction. However, the flows are different, neither the primed and unprimed measures nor their OS data coincide at any finite resolution scale $M$. Indeed, the path integral flow produces, if it exists, a fixed point family of measures $M \mapsto \mu_{R M}^{*}$ which qualify as the cylindrical projections, corresponding to the resolutions $M$, of a continuum measure $\mu_{R}^{*}$. This continuum measure can be obtained by computing the continuum limit $\mu_{R}^{*}=\lim _{M \rightarrow \infty} \mu_{R M}^{*}$. On the other hand, the Wiener measures $\mu_{R M}^{\prime *}$ corresponding to a fixed point family of OS data $\left(\mathcal{H}_{R M}^{\prime *}, \Omega_{R M}^{* *}, H_{R M}^{* *}\right)$ which is obtained via the direct Hamiltonian flow do not satisfy the cylindrical consistency condition. However, one can compute the corresponding continuum limit $\mu_{R}^{\prime *}=\lim _{M \rightarrow \infty} \mu_{R}^{\prime *}$. If in fact $\mu_{R}^{*}=\mu_{R}^{\prime *}$ then the continuum limit of the fixed point of OS data are the OS data of $\mu_{R}^{*}$ and thus the two different flows still produce the same continuum theory.

In our companion paper [57] we will demonstrate that the two flows indeed produce the same continuum theory for the case of a free, massive scalar field in one spatial dimension. Ideally one would like to have necessary and/or sufficient criteria at one's disposal, which would guarantee such a coincidence in the general case. These criteria may impose restrictions on the class of coarse graining maps, discretisations, field content and Hamiltonians. However, even if such criteria cannot be found or if there are examples for which the continuum limits of the fixed points of the flows do not coincide, still the direct Hamiltonian flow may produce a continuum Hamiltonian whose finite resolution matrix elements have much improved properties as compared the naive discretisations that started the direct renormalisation flow. Thus, the stage is prepared to attack more difficult models and eventually background independent quantum gravity in order to remove (or rather fix point) the quantisation ambiguities of the quantum dynamics $[63,78-90]$. In that respect note that we were careful to state all the ingredients of the renormalisation flow in a background independent way, even if the tests we performed are in the context of free scalar QFT in Minkowski space.

\section{Acknowledgements}

Part of this work was financially supported by a grant from the Friedrich-Alexander University to the 
Emerging Fields Project "Quantum Geometry" under its Emerging Fields Initiative. K. L. thanks the German National Merit Foundation for financial support. T.L. thanks the Heinrich-Böll Foundation for financial support.

\section{A Inductive Limits}

Here we just list elementary definitions and implications on the topic of inductive limits of Hilbert spaces and operators.

\section{Definition.}

Let $I$ be an index set partially ordered and directed by $<$.

$i$.

A system of Hilbert spaces $\left\{\mathcal{H}_{i}\right\}_{i \in I}$ is called an inductive family iff for each $i<j$ there exist isometric injections

$$
J_{i j}: \mathcal{H}_{i} \rightarrow \mathcal{H}_{j}, J_{i i}=\mathrm{id}_{\mathcal{H}_{i}}
$$

that are subject to the compatibility condition for each $i<j<k$

$$
J_{i k}=J_{j k} J_{i j}
$$

ii.

A family of operators $\left\{A_{i}\right\}_{i \in I_{c}}$ with dense domains $D_{i}$ for $i \in I_{c} \subset I$ co-final in $I$ (i.e. for each $i \in I$ there exists $j \in I_{c}$ such that $i<j$ ) is said to be an inductive system provided that for each $i<j$ both in $I_{c}$

$$
J_{i j} D_{i} \subset D_{j}, \quad A_{j} J_{i j}=J_{i j} A_{i}
$$

\section{Lemma.}

$i$.

An inductive system of Hilbert spaces has an inductive limit, i.e. there exists a Hilbert space $\mathcal{H}$ and isometric injections $J_{i}: \mathcal{H}_{i} \rightarrow \mathcal{H}$ for each $i \in I$ such that for all $i<j$

$$
J_{j} J_{i j}=J_{i}
$$

The inductive limit Hilbert space is unique up to a unitary map.

ii.

An inductive system of operators has an inductive limit, i.e. there exists an operator $A$ densely defined on a domain $D \subset \mathcal{H}$ where $\mathcal{H}$ is the inductive limit Hilbert space such that for each $i \in I_{c}$

$$
J_{i} A_{i}=A J_{i}
$$

If moreover $A_{i}$ is essentially self-adjoint with core $D_{i}$ then so is $A$ on $D$.

Proof:

i.

We define vectors $\psi_{i} \in \mathcal{H}_{i}, \psi_{j} \in \mathcal{H}_{j}$ to be equivalent iff for some and therefore any ${ }^{6} i, j<k$ we have $J_{i k} \psi_{i}=J_{j k} \psi_{j}$. We consider the equivalence classes $\left[\psi_{i}\right]$ and equip them with the inner product

$$
\left\langle\left[\psi_{i}\right],\left[\psi_{j}\right]\right\rangle:=\left\langle J_{i k} \psi_{i}, J_{k j} \psi_{j}\right\rangle_{\mathcal{H}_{k}}
$$

where $k$ is any $i, j<k$. This is independent of the representative because for any $i, j<k^{\prime}$ we find $k, k^{\prime}<\hat{k}$ and have by isometry and consistency

$$
\begin{aligned}
\left\langle J_{i k} \psi_{i}, J_{j k} \psi_{j}\right\rangle & =\left\langle J_{k \hat{k}} J_{i k} \psi_{i}, J_{k \hat{k}} J_{j k} \psi_{j}\right\rangle=\left\langle J_{i \hat{k}} \psi_{i}, J_{j \hat{k}} \psi_{j}\right\rangle= \\
& =\left\langle J_{k^{\prime} \hat{k}} J_{i k^{\prime}} \psi_{i}, J_{k^{\prime} \hat{k}} J_{j k^{\prime}} \psi_{j}\right\rangle=\left\langle J_{i k^{\prime}} \psi_{i}, J_{j k^{\prime}} \psi_{j}\right\rangle
\end{aligned}
$$

\footnotetext{
${ }^{6}$ Suppose $k^{\prime}>i, j$ then we find $\hat{k}>k, k^{\prime}$ and have $0=J_{k \hat{k}}\left(J_{i k} \psi_{i}-J_{j k} \psi_{j}\right)=J_{i \hat{k}} \psi_{i}-J_{j \hat{k}} \psi_{j}=J_{k^{\prime} \hat{k}}\left(J_{i k^{\prime}} \psi_{i}-\right.$ $J_{j k^{\prime}} \psi_{j}$ ) whence by injectivity $J_{i k^{\prime}} \psi_{i}=J_{j k^{\prime}} \psi_{j}$.
} 
We extend the scalar product to the space of the formal finite linear combinations of equivalence classes by sesquilinearity and complete it to obtain the inductive limit Hilbert space $\mathcal{H}$. The required maps are

$$
J_{i} \psi_{i}:=\left[\psi_{i}\right]
$$

and for any $i<j$ we have

$$
J_{j} J_{i j} \psi_{i}=\left[J_{i j} \psi_{i}\right]=\left[\psi_{i}\right]=J_{i} \psi_{i}
$$

since the vectors $\psi_{i}, J_{i j} \psi_{i}$ are equivalent (choose $k=j>i, j$ ). The $J_{i}$ are injections since $J_{i} \psi_{i}=$ $\left[\psi_{i}\right]=0$ means that $J_{i j} \psi_{i}=0$ for some $i<j$ hence $\psi_{i}=0$. They are isometric since (pick $k=j>i)$.

$$
\left\langle J_{i} \psi_{i}, J_{i} \psi_{i}^{\prime}\right\rangle_{\mathcal{H}}=\left\langle\left[\psi_{i}\right],\left[\psi_{i}^{\prime}\right]\right\rangle_{\mathcal{H}}=\left\langle\psi_{i}, \psi_{i}^{\prime}\right\rangle_{\mathcal{H}_{i}}
$$

Finally suppose that two inductive limits $\left(\mathcal{H},\left\{J_{i}\right\}_{i \in I}\right)$ and $\left(\mathcal{H}^{\prime},\left\{J_{i}^{\prime}\right\}_{i \in I}\right)$ have been found. We define

$$
U: \mathcal{H} \rightarrow \mathcal{H}^{\prime} ; J_{i} \psi_{i} \mapsto J_{i}^{\prime} \psi_{i}
$$

and extend by linearity to the dense domain of the finite linear combinations of the $J_{i} \psi_{i}$. It has the inverse on its image

$$
U^{-1}: \mathcal{H}^{\prime} \rightarrow \mathcal{H} ; \quad J_{i}^{\prime} \psi_{i} \mapsto J_{i} \psi_{i}
$$

and is isometric there (pick any $i, j<k$ )

$$
\left\langle U J_{i} \psi_{i}, U J_{j} \psi_{j}\right\rangle_{\mathcal{H}^{\prime}}=\left\langle J_{i}^{\prime} \psi_{i}, J_{j}^{\prime} \psi_{j}\right\rangle_{\mathcal{H}^{\prime}}=\left\langle J_{k}^{\prime} J_{i k} \psi_{i}, J_{k}^{\prime} J_{j k} \psi_{j}\right\rangle_{\mathcal{H}^{\prime}}=\left\langle J_{i k} \psi_{i}, J_{j k} \psi_{j}\right\rangle_{\mathcal{H}_{k}}=\left\langle J_{i} \psi_{i}, J_{j} \psi_{j}\right\rangle_{\mathcal{H}}
$$

and can therefore be extended to a unitary operator to all of $\mathcal{H}$ by continuity.

ii.

We define $D$ to be the finite linear combinations of the vectors $J_{i} \psi_{i}, \psi_{i} \in D_{i}$ with $i \in I_{c}$. For $i \notin I_{c}$ we find $i<j \in I_{c}$ such that $J_{i} \psi=J_{j} J_{i j} \psi_{i}$, so that it is enough to define $A$ on the $J_{i} \psi_{i}, i \in I_{c}$. As the $D_{i}$ are dense in $\mathcal{H}_{i}, D$ is dense in $\mathcal{H}$. Then we define for $i \in I_{c}$

$$
A J_{i} \psi_{i}:=J_{i} A_{i} \psi_{i}
$$

and extend by linearity.

This definition is consistent for suppose that $J_{i} \psi_{i}=J_{j} \psi_{j}$ then we find $i, j<k$ all in $I_{c}$ such that $J_{i k} \psi_{i}=J_{j k} \psi_{j}$ and

$$
A\left(J_{i} \psi_{i}-J_{j} \psi_{j}\right)=A\left(J_{k} J_{i k} \psi_{i}-J_{k} J_{j k} \psi_{j}\right)=J_{k} A_{k}\left(J_{i k} \psi_{i}-J_{j k} \psi_{j}\right)=0
$$

Finally, by the basic criterion of essential self-adjointness, we know that $\left(A_{j} \pm i 1_{\mathcal{H}_{j}}\right) D_{j}$ is dense in $\mathcal{H}_{j}$. It follows that for any $j \in I_{c}$

$$
\left[A \pm i 1_{\mathcal{H}}\right] J_{j} D_{j}=J_{j}\left[A_{j} \pm i 1_{\mathcal{H}_{j}}\right] D_{j}
$$

is dense in $J_{j} D_{j}$, hence $\left[A \pm i 1_{\mathcal{H}}\right] D$ is dense in $\mathcal{H}$ and $A$ is essentially self-adjoint.

\section{B Notes on Nelson-Symanzik Positivity}

In the main text we claimed that (2.8) implies (2.7). We sketch the proof given in $[7,8]$ and adapt it to our notation. For all $t \in \mathbb{R}$, let $Q_{t}=\Delta(\mathfrak{B})$ and $Q=\times_{t \in \mathbb{R}} Q_{t}$ and let $\Sigma$ be the Baire sets of $Q$, i.e. the smallest $\sigma$-algebra, such that all compactly supported continuous functions are measurable. We will construct $\mu$ as a probability measure on $(Q, \Sigma)$. By the Gel'fand isomorphism, we have $\mathfrak{B} \simeq C(\Delta(\mathfrak{B})) \simeq L^{\infty}(\Delta(\mathfrak{B}), \mathrm{d} \nu)$. For $f \in L^{2}(\Delta(\mathfrak{B}), \mathrm{d} \nu)$, the positivity criterion guarantees that $e^{-\beta H}$ is positivity preserving, i.e. $f \geq 0$ implies $e^{-\beta H} f \geq 0$. Since $e^{-\beta H}$ is a contraction on $L^{2}(\Delta(\mathfrak{B}), \mathrm{d} \nu)$, by the argument in [8], the preservation of positivity ensures that it is also a contraction on $L^{1}(\Delta(\mathfrak{B}), \mathrm{d} \nu)$. By duality, we obtain a corresponding positivity preserving contraction 
on $L^{\infty}(\Delta(\mathfrak{B}), \mathrm{d} \nu)$, which defines a map $A_{t}$ on $C(\Delta(\mathfrak{B}))$ by isomorphism. In order to proceed, we quote the following lemma from [7]: Let $Y_{1}, \ldots, Y_{n}$ be compact Hausdorff spaces and let $\mu_{n}$ be a Baire measure on $Y_{n}$. Moreover, for $i=1, \ldots, n-1$, let $X_{i}$ be a bounded, positivity preserving map from $C\left(Y_{i}\right)$ to $C\left(Y_{i+1}\right)$ such that $X_{i} 1=1$. Then there exists a unique measure $\hat{\nu}$ on $Y_{1} \times \ldots \times Y_{n}$ such that for any $f_{1}, \ldots, f_{n}$ in $C\left(Y_{1}\right), \ldots, C\left(Y_{n}\right)$, we have

$$
\int f_{1}\left(y_{1}\right) \ldots f_{n}\left(y_{n}\right) \mathrm{d} \hat{\nu}=\int f_{n} X_{n-1}\left(f_{n-1} X_{n-2}\left(f_{n-2} \ldots f_{2} X_{1} f_{1}\right) \ldots\right) \mathrm{d} \nu_{n} .
$$

Given $t_{1}<\ldots<t_{n}$, we apply this lemma to $\mu_{n}=\nu, Y_{i}=Q_{t_{i}}=\Delta(\mathfrak{B})$ and $X_{i}=A_{\left|t_{i+1}-t_{i}\right|}$ in order to obtain measures $\mu_{t_{1}, \ldots, t_{n}}$ on $Q_{t_{1}} \times \ldots \times Q_{t_{n}}$. These measures can be used to define the measure $\mu$ on $(Q, \Sigma)$ by first defining it for functions $F$ in $C(Q)$ that depend only on finitely many $q_{t_{i}} \in Q_{t_{i}}$ :

$$
\int F \mathrm{~d} \mu:=\int F\left(q_{t_{1}}, \ldots, q_{t_{n}}\right) \mathrm{d} \mu_{t_{1}, \ldots, t_{n}}
$$

This constitutes a positive linear functional on the set $\bigcup_{n} \bigcup_{t_{1}, \ldots, t_{n}} C\left(Q_{t_{1}} \times \ldots \times Q_{t_{n}}\right.$, which lies dense in $C(Q)$ by the Stone-Weierstrass theorem. The functional thus extends to $C(Q)$ and by the Riesz-Markov theorem, we obtain a unique measure $\mu$ on $(Q, \Sigma)$. By construction, the positivity preservation guarantees that $\mu$ is a positive measure and since $\mu(Q)=\int_{\Delta(\mathfrak{B})} \mathrm{d} \nu=\langle\Omega, \Omega\rangle=1$, it is a probability measure.

\section{References}

[1] J. Glimm and A. Jaffe, "Quantum Physics - A functional integral Point of View", SpringerVerlag, New York, (1987)

[2] Juerg Froehlich, "An introduction to some topics in Constructive QFT", Springer-Verlag, New York, (1978)

[3] Vincent Rivasseau, "Constructive Field Theory and Applications: Perspectives and Open Problems", J. Math. Phys. 41 (2000) 3764-3775

[4] Barry Simon. The P $(\phi) 2$ Euclidean (Quantum) Field Theory. Princeton Unviersity Press (1974)

[5] K. Osterwalder and R. Schrader, "Axioms for Euclidean Green's Functions", Commun. math. Phys. 31 (1973) 83-112

[6] K. Osterwalder, E. Seiler. Gauge Field Theories on a Lattice. Annals of Physics 110 (1978)

[7] Barry Simon. Positivity of the Hamiltonian Semigroup and the Construction of Euclidean Region Fields. Helvetica Physics Acta 46 (1973)

[8] Barry Simon, Raphael Hegh-Krohn. Hypercontractive semigroups and two dimensional selfcoupled Bose fields. Journal of Functional Analysis 9 (1972) 121-180.

[9] Yasuo Yamasaki. Kolmogorov's Extension Theorem for Infinite Measures. Publ. RIMS, Kyoto Univ 10 (1975)

[10] A. Ashtekar, D. Marolf, J. Mourao, T. Thiemann. Osterwalder-Schrader Reconstruction and Diffeomorphism Invariance. Class.Quant.Grav. 17 (2000) [arXiv:quant-ph/9904094]

[11] M. Henneaux, C. Teitelboim. Quantization of Gauge Systems. Princeton University Press (1994)

[12] A. S. Vytheeswaran. Gauge unfixing in second class constrained systems. Annals Phys. 236 (1994) 297-324.

[13] P. Mitra, R. Rajaraman. Gauge-invariant reformulation of an anomalous gauge theory. Physics Letters B 225 (1989) 267-271.

R. Anishetty, A. S. Vytheeswaran. Gauge invariance in second-class constrained systems. Journal of Physics A: Mathematical and General 26 (1993), no. 20 5613-5619.

[14] Stephen Fulling. Aspects of Quantum Field Theory in Curved Spacetime. Cambridge University Press (1989)

[15] Tadeusz Balaban, "Large field renormalization I. The Basic Step of the R Operation", Commun. math. Phys. 122 (1989) 207-263 
[16] Tadeusz Balaban, "Large field renormalization II. Localization, Exponentation, and Bounds for the R. Operation", Commun. math. Phys. 122 (1989) 355-392

[17] J. Dimock. The Renormalization Group According to Balaban I - Small Fields. Rev. Math. Phys. 25 (2012) [arXiv:1108.1335]

[18] J. Dimock. The Renormalization Group According to Balaban II - Large Fields. Journal of Mathematical Physics 54 (2012) [arXiv:1212.5562]

[19] J. Dimock. The Renormalization Group According to Balaban III - Convergence. J. Ann. Henri Poincaré 15: 2133. https://doi.org/10.1007/s00023-013-0303-3 (2014) [arXiv:1304.0705]

[20] J. Dimock. Quantum Mechanics and Quantum Field Theory. Cambridge University Press, Cambridge, 2011.

[21] A. S. Wightman (eds.) A. S. Wightman (auth.), G. Velo. Constructive Quantum Field Theory II. NATO ASI Series 234. Springer US, 1 edition, 1990.

[22] A. S. Wightman (eds.) Arthur S. Wightman (auth.), G. Velo. Fundamental Problems of Gauge Field Theory. NATO ASI Series 141. Springer US, 1 edition, 1986.

[23] T. Balaban. Recent results in constructing gauge fields. Physica A: Statistical Mechanics and its Applications, 124, 1984.

[24] Alan David Sokal. A alternate constructive approach to the phi -4(3) quantum field theory, and a possible destructive approach to phi-4(4). 011981.

[25] K. Gawedzki; A. Kupiainen. A rigorous block spin approach to massless lattice theories. Communications in Mathematical Physics, 77, 1980.

[26] A. Jaffe; H. Lehmann; P. K. Mitter; I. M. Singer; R. Stora (eds.); Luis Alvarez-Gaum (auth.); G. $t$ Hooft. Progress in Gauge Field Theory. NATO ASI Series 115. Springer US, 1 edition, 1984.

[27] David Brydges; Jrg Frhlich; Erhard Seiler. On the construction of quantized gauge fields. i. general results. Annals of Physics, 121, 1979.

[28] E. Seiler. Gauge theories as a problem of constructive QFT and statistical mechanics. LNP0159. Springer, 1 edition, 1982.

[29] H. J. Borchers; J. Yngvason. Necessary and sufficient conditions for integral representations of wightman functionals at schwinger points. Communications in Mathematical Physics, 47, 1976.

[30] K.G. Wilson and J. Kogut. The renormalization group and the $\epsilon$ expansion. Physics Reports $12(1974)$

[31] T.L. Bell and K.G. Wilson. Nonlinear renormalization groups. Phys. Rev. B 10 (1974)

[32] Kenneth G. Wilson. The renormalization group: Critical phenomena and the Kondo problem. Rev. Mod. Phys 47 (1975)

[33] A. Corichi, T. Vukasinac, J.A. Zapata. Hamiltonian and physical Hilbert space in polymer quantum mechanics. Class. Quant. Grav. 24 (2007)

[34] E. Manrique, R. Oeckl, A. Weber and J.A. Zapata. Loop quantization as a continuum limit. Class. Quant. Grav. 23 (2006)

[35] B. Bahr, B Dittrich. Improved and Perfect Actions in Discrete Gravity. Phys Rev D 90 (2009)

[36] B. Bahr, B. Dittrich, S. Steinhaus. Perfect discretization of reparametrization invariant path integrals Phys. Rev. D 83 (2011)

[37] J. Geloun, E. Livine. Some classes of renormalizable tensor models. J. Math. Phys.54 (2013)

[38] B. Dittrich, S. Mizera, S. Steinhaus. Decorated tensor network renormalization for lattice gauge theories and spin foam models. New J. Phys 18 (2016)

[39] G. Evenbly, G. Vidal. Tensor Network Renormalization. Phys Rev. Lett 115 (2015)

[40] B Bahr, S. Steinhaus. Hypercuboidal renormalization in spin foam quantum gravity. Phys Rev D 95 (2017)

[41] S. D. Glazek, K. G. Wilson. Perturbative Renormalisation Group for Hamiltonians. Phys Rev D 49 (1994)

[42] F. J. Wegner. Phys Rev B 5, 6 (1972)

[43] G. Alexanian, E. F. Moreno. On the renormalization of Hamiltonians. Phys Lett B 450 (1999)

[44] G. Ciobanu, V. Barsan, A.T. Mincu. Hamiltonian renormalization with applications to the quantum quartic oscillator. Rom J Phys 55 (2010) 
[45] J. Kijowski, A Okolow. A modification of the projective construction of quantum states for field theories. J. Math Phys.58 (2016)

[46] S. Lanéry, T. Thiemann. Projective Limits of State Spaces I.Classical Formalism. J. Geometry Phys.111 (2017)

S. Lanéry, T. Thiemann. Projective Limits of State Spaces )II.Quantum Formalism J. Geometry Phys.116 (2017)

S. Lanéry, T. Thiemann. Projective Limits of State Spaces III.Toy-Models [arXiv:1411.3591]

S. Lanéry, T. Thiemann. Projective Limits of State Spaces IV.Fractal Label Sets [arXiv:1510.01926]

[47] Suzanne Lanéry. Projective Limits of State Spaces: QFT without a Vacuum. [arXiv:1604.05629]

[48] A. Milsted, T. Osborne. Quantum Yang-Mills theory: an overview of a programme. Phys. Rev. D 98 (2018) [arXiv:1604.01979]

[49] A. Ashtekar. Lectures on non perturbative canonical gravity. Word Scientifitc (1991)

[50] C. Rovelli. Quantum Gravity, (Cambridge University Press, Cambridge, 2004).

[51] Thiemann. Modern Canonical Quantum General Relativity, (Cambridge University Press, Cambridge, 2007). [gr-qc/0110034]

[52] K. Giesel and H. Sahlmann. From Classical To Quantum Gravity: Introduction to Loop Quantum Gravity. (2013), [arXiv:1203.2733v2]

[53] M. Reuter, F. Saueressig. Functional Renormalization Group Equations, Asymptotic Safety and Quantum Einstein Gravity. [arXiv:0708.1317]

[54] R. Percacci. A short introduction to asymptotic safety. [arXiv:1110.6389]

[55] M. Reuter. F. Saueressig. Quantum Einstein Gravity. New Journal of Physics 14 (2012)

[56] Astrid Eichhorn. Status of the asymptotic safety paradigm for QG an matter [arXiv:1709.03696]

[57] Thorsten Lang, Klaus Liegener, Thomas Thiemann. Hamiltonian Renormalisation II. Renormalisation Flow of $1+1$ dimensional free, scalar quantum fields: Derivation. Class.Quant.Grav. 35 24, 245012 (2018) [arXiv:1711.06727]

[58] Thorsten Lang, Klaus Liegener, Thomas Thiemann. Hamiltonian Renormalisation III. Renormalisation Flow of $1+1$ dimensional free, scalar quantum fields: Properties. Class. Quant.Grav. 35 24, 245013 (2018) [arXiv:1711.05688]

[59] Thorsten Lang, Klaus Liegener, Thomas Thiemann. Hamiltonian Renormalisation IV. Renormalisation Flow of $\mathrm{D}+1$ dimensional free, scalar quantum fields and Rotation Invariance. Class.Quant.Grav. 35 24, 245014 (2018) [arXiv:1711.05695]

[60] K. V. Kuchar, C. G. Torre. Gaussian reference fluid and interpretation of quantum geometrodynamics. Phys. Rev. D43 (1991) 419-441.

[61] J. Brown and K. Kuchař. Dust as a standard of space and time in canonical quantum gravity. Phys. Rev. D51 (1995), 5600-5629. [gr-qc/9409001]

[62] V. Husain and T. Pawlowski. Time and a physical Hamiltonian for a quantum gravity. Phys. Rev. Lett. 108 (2011) [arXiv:1108.1145v2]

[63] K. Giesel and T Thiemann. Scalar Material Reference Systems and Loop Quantum Gravity. Class. Quant. Grav. 32 (2015) [arXiv:1206.3807v2]

[64] Walter Thirring. A Course in Mathematical Physics 3 Springer-Verlag (1981)

[65] O. Bratteli, D.W. Robinson. Operator Algebras and Quantum Statistical Mechanics 1 \& 2. Springer Verlag (2003-2004)

[66] A. Ashtekar. Old problems in the light of new variables. Contemporary Math. 71 (1988)

[67] J. F. G. Barbero. A real polynomial formulation of general relativity in terms of connection Phys. Rev. D 49 (1994)

[68] J. Fernando Barbero. Real ashtekar variables for lorentzian signature space times Phys Rev. D 51 (1995)

[69] C. Fleischhack. A new type of loop independence and SU(N) quantum Yang-Mills theory in two-dimensions. J. Math Phys.40 (1999)

[70] C. Fleischhack. Representations of the Weyl Algebra in quantum geometry. Commun. Math. Phys 249 (2004)

[71] Rudolf Haag. Local Quantum Physics. Springer-Verlag (1992) 
[72] M. Reed, B. Simon. Methods of modern mathematical physics. I-IV Academica Press, In. (1980)

[73] Walter Rudin. Real and Complex analysis. McGraw-Hill Book Company (1987)

[74] R. Fernandez, J. Frhlich, A.D. Sokal. Random Walks, Critical Phenomena, and Triviality in QFT. Springer-Verlag (1992)

[75] Peter Hasenfratz. Prospects for perfect actions. Nucl. Phys. Proc. Suppl. 63 (1998)

[76] Peter Hasenfratz. The theoretical background and properties of perfect actions. (2008) [arXiv:hep-lat/9803027]

[77] P. Hasenfratz and F. Niedermayer. Perfect lattice action for asymptotically free theories. Nucl Phys B 414 (1993)

[78] T. Thiemann. Quantum Spin Dynamics (QSD). Class. Quantum Grav. 15 (1998), 839-873. [gr-qc/9606089]

[79] T. Thiemann . Quantum Spin Dynamics (QSD) II. Class. Quantum Grav.15 (1998), 875-905. [gr-qc/9606090]

[80] T. Thiemann. Quantum Spin Dynamics (QSD): V. Quantum gravity as the natural regulator of the Hamiltonian constraint of matter quantum field theories. Class. Quantum Grav. 15 (1998), 1281-1314. [gr-qc/9705019]

[81] T. Thiemann. The Phoenix project: master constraint programme for loop quantum gravity. Class. Quant. Grav. 23 (2006), 2211-2248. [gr-qc/0305080]

[82] K. Giesel and T. Thiemann. Algebraic quantum gravity (AQG) I. Conceptual setup. Class. Quant. Grav. 24 (2007) 2465-2498. [gr-qc/0607099]

K. Giesel and T. Thiemann. Algebraic quantum gravity (AQG) II. Semiclassical analysis. Class. Quant. Grav. 24 (2007) 2499-2564. [gr-qc/0607100]

K. Giesel and T. Thiemann. Algebraic quantum gravity (AQG) III. Semiclassical perturbation theory. Class. Quant. Grav. 24 (2007) 2565-2588. [gr-qc/0607101]

K. Giesel and T. Thiemann. Algebraic quantum gravity (AQG). IV. Reduced phase space quantisation of loop quantum gravity. Class. Quant. Grav. 27 (2010) 175009. [gr-qc/0711.0119]

[83] K. Liegener, T. Thiemann. Towards the fundamental spectrum of the Quantum Yang-Mills Theory. Phys Rev D.94 (2016)

[84] T. Thiemann. Gauge Field Theory Coherent States (GCS): I. General Properties. Class. Quant. Grav. 18 (2001)

[85] T. Thiemann, O. Winkler. Gauge Field Theory Coherent States (GCS): II. Peakedness Properties. Class. Quant. Grav. 18 (2001)

[86] T. Thiemann, O. Winkler. Gauge Field Theory Coherent States (GCS): III. Ehrenfest Theorems. Class. Quant. Grav. 18 (2001)

[87] H. Sahlmann, T. Thiemann, O. Winkler. Coherent states for canonical quantum general relativity and the infinite tensor product extension. Nucl. Phys. B 606 (2001)

[88] A. Dapor, K. Liegener. Cosmological Effective Hamiltonian from full LQG Dynamics. Phys.Lett. B 785 (2018) 506-510 [arXiv:1706.09833]

[89] A. Dapor, K. Liegener. Cosmological Coherent State Expectation Values in LQG I Isotropic Kinematics. Class. Quant. Grav. 35 (2018) [arXiv:1710.04015]

[90] T. Thiemann A length operator for canonical quantum gravity. J. Math Phys 39 (1998) 